\title{
Article
}

\section{Global Distribution of the Reniform Nematode Genus Rotylenchulus with the Synonymy of Rotylenchulus macrosoma with Rotylenchulus borealis}

\author{
Juan E. Palomares-Rius ${ }^{1}$, Ilenia Clavero-Camacho ${ }^{1}$, Antonio Archidona-Yuste ${ }^{2}{ }^{\mathbb{D}}$, \\ Carolina Cantalapiedra-Navarrete ${ }^{1}$, Guillermo León-Ropero ${ }^{1}$, Sigal Braun Miyara ${ }^{3}$, Gerrit Karssen ${ }^{4,5}$ \\ and Pablo Castillo ${ }^{1, *(1)}$
}

1 Instituto de Agricultura Sostenible (IAS), Consejo Superior de Investigaciones Científicas (CSIC), Avda, Menéndez Pidal s/n, 14004 Córdoba, Spain; palomaresje@ias.csic.es (J.E.P.-R.); iclavero@ias.csic.es (I.C.-C.); ccantalapiedra@ias.csic.es (C.C.-N.); gleon@ias.csic.es (G.L.-R.)

2 Helmholtz Centre for Environmental Research-UFZ, Department of Ecological Modelling, Permoserstrasse 15, 04318 Leipzig, Germany; antonio.archidona-yuste@ufz.de

3 Nematology and Chemistry Units, Agricultural Research Organization (ARO), The Volcani Center, Department of Entomology, Bet Dagan 50250, Israel; sigalhor@volcani.agri.gov.il

4 Nematology Research Unit, Department of Biology, Ghent University, K.L. Ledeganckstraat 35, 9000 Ghent, Belgium; gerrit.karssen@ugent.be

5 National Plant Protection Organization, Wageningen Nematode Collection, P.O. Box 9102, 6700 HC Wageningen, The Netherlands

* Correspondence: p.castillo@csic.es

\section{check for} updates

Citation: Palomares-Rius, J.E.; ClaveroCamacho, I.; Archidona-Yuste, A.; Cantalapiedra-Navarrete, C.; LeónRopero, G.; Braun Miyara, S.; Karssen, G.; Castillo, P. Global Distribution of the Reniform Nematode Genus Rotylenchulus with the Synonymy of Rotylenchulus macrosoma with Rotylenchulus borealis. Plants 2021, 10, 7 . https://dx.doi.org/10.3390/plants 10010007

Received: 24 November 2020 Accepted: 21 December 2020 Published: 23 December 2020

Publisher's Note: MDPI stays neutral with regard to jurisdictional claims in published maps and institutional affiliations.

Copyright: () 2020 by the authors. Licensee MDPI, Basel, Switzerland. This article is an open access article distributed under the terms and conditions of the Creative Commons Attribution (CC BY) license (https: / / creativecommons.org/ licenses/by/4.0/).

\begin{abstract}
Reniform nematodes of the genus Rotylenchulus are semi-endoparasites of numerous herbaceous and woody plant roots that occur largely in regions with temperate, subtropical, and tropical climates. In this study, we compared 12 populations of Rotylenchulus borealis and 16 populations of Rotylenchulus macrosoma, including paratypes deposited in nematode collections, confirming that morphological characters between both nematode species do not support their separation. In addition, analysis of molecular markers using nuclear ribosomal DNA (28S, ITS1) and mitochondrial DNA (coxI) genes, as well as phylogenetic approaches, confirmed the synonymy of $R$. macrosoma with $R$. borealis. This study also demonstrated that $R$. borealis (= macrosoma) from Israel has two distinct rRNA gene types in the genome, specifically the two types of $D 2-D 3$ (A and B). We provide a global geographical distribution of the genus Rotylenchulus. The two major pathogenic species (Rotylenchulus reniformis and Rotylenchulus parvus) showed their close relationship with warmer areas with high annual mean temperature, maximum temperature of the warmest month, and minimum temperature of the coldest month. The present study confirms the extraordinary morphological and molecular diversity of $R$. borealis in Europe, Africa, and the Middle East and comprises a paradigmatic example of remarkable flexibility of ecological requirements within reniform nematodes.
\end{abstract}

Keywords: Bayesian inference; cytochrome c oxidase subunit 1; distribution; D2-D3 expansion domains of $28 S$ rRNA gene; ITS1; phylogeny

\section{Introduction}

Reniform nematodes of the genus Rotylenchulus are an economically important polyphagous group of highly adapted obligate plant parasites that parasitize numerous plants and crops usually associated with temperate, subtropical, and tropical climates [1]. The genus Rotylenchulus Linford and Oliveira [2] comprise 11 valid species; some of them are distributed worldwide, whereas others have shown a limited distribution $[1,3,4]$. This genus has been reported in 77 countries of Africa, Asia, Europe, North and South America, and Australia $[1,3,4]$. The influence of future global climate change could shorten the life cycle of these nematodes and may expand the distribution of well-adapted species to 
drought conditions [5,6]. However, other factors such as the low population density in soil, no apparent harvest losses in some crops, or the difficulties for an accurate identification for some Rotylenchulus species could thwart their precise geographical distribution. For these reasons, Rotylenchulus spp. could be regarded as a "neglected pathogen", but also as a potentially dangerous pathogen in the future because of new ecological conditions predicted in global climate change scenarios [7]. Consequently, an updating of the global distribution of this group of nematodes allowed us to know the climatic conditions adapted to each species, which are essential to predict the response of this genus to climate change [8,9].

Rotylenchulus spp. show high intraspecific variability of some morphological diagnostic features in immature females (the developmental stage usually employed for species identification) [3], and for this reason, it is necessary to use molecular markers for precise species identification. In this regard, the use of rRNA markers is challenging due to the previously noted presence of several gene copies that are not well homogenized in the genome, and for this reason, several different amplicon sizes and associated sequences can be observed [4]. A prominent example of this high intraspecific variability was established in the study on several populations of Rotylenchulus macrosoma by Dasgupta et al. [10] and $R$. borealis by Loof and Oostenbrink [11].

In 1952, Oostenbrink found a population of reniform-shaped nematode in a soil sample from Arnhem (The Netherlands). Subsequent examination and comparison with published descriptions showed that the new nematode represented an undescribed species, proposed as Rotylenchulus borealis Loof \& Oostenbrink [11], referring to its occurrence in northern countries, since the other species of the genus were mainly known from the tropical and subtropical regions [11]. Some years later, Dasgupta et al. [10] revised the genus Rotylenchulus and described a new species from olive in Hulda, Israel, closely related to $R$. borealis, named Rotylenchulus macrosoma (original spelling macrosomus). $R$. macrosoma differed from the former by its larger body length of immature females and males (0.52-0.64 mm, $0.50-0.68 \mathrm{~mm}$ vs. $0.37-0.46 \mathrm{~mm}, 0.40-0.49 \mathrm{~mm}$ in $R$. borealis, respectively), larger female stylet (18-22 vs. 13-16 $\mu \mathrm{m}$ in R. borealis), and longer hyaline portion of immature female tail ( $\mathrm{h}=13-18 \mathrm{vs}$. $9-13 \mu \mathrm{m}$ in $R$. borealis). These limited differences between both species have been confirmed by posterior morphometrics of several African populations studied by Germani [12], as well as the recent $R$. macrosoma populations studied from Europe [3,9].

In 2003, Castillo et al. [13] detected a population of reniform nematodes infecting the roots of wild olive trees (Olea europea L. ssp. sylvestris) on a sandy soil in Cádiz province, southern Spain, which was identified as R. macrosoma. Morphometric of the Spanish population agreed with the original description of R. macrosoma, except for a shorter stylet length (15-18 vs. 18-22 $\mu \mathrm{m}$ ), which was considered as an intraspecific variability. Later on, in 2016, Van den Berg et al. [3] provided morphological and molecular characterization of 6 out of 11 presently known species of Rotylenchulus, including three Spanish populations (two and one from Cádiz and Seville provinces, respectively) of R. macrosoma, which formed a separate and well-supported clade within phylogenetic trees of D2-D3 expansion segments of $28 S$ rRNA, ITS, and hsp 90 genes [3]. This study also reported high levels of intraspecific and intra-individual variations of rRNA with two or more distinct types of rRNA genes, namely, type A and B [3]. These phylogenetic relationships were confirmed by posterior studies on additional new reports of $R$. macrosoma populations from several European countries including the Czech Republic, France, Germany, Greece, Hungary, Italy, Portugal, Romania, Serbia, and Spain [4,9]. In a recent study on the integrative characterization of plant-parasitic nematodes of potato in Rwanda, Niragire [14] provided morphological and molecular data of a population of R. macrosoma from Burera (North Rwanda), but no sequences were deposited in the National Center for Biotechnology Information (NCBI) database. Molecular data available for R. borealis is a $28 \mathrm{~S}$ rRNA sequence obtained from a Belgian population (MK558206) and the mentioned sequence for Burera clustered together with the Spanish R. macrosoma populations [14]. However, this Belgian population (Oudenaarde, Belgium) of $R$. borealis was not mentioned in the 
associated paper with the NCBI sequence and no morphological data were available alongside it [15]. This sequence has a 99.45\% identity with $R$. macrosoma-KT003748 from Spain. Recently, Qing et al. [16] studied the rRNA variation (intragenomic polymorphism) across 30 terrestrial nematode species and sequenced 28S and ITS1 from a population of $R$. macrosoma in Israel, which clustered together in the same clade with $R$. macrosoma populations from Spain and Crete (Greece) and clearly separated from other Rotylenchulus spp. Finally, in the last months, one new $28 S$ rRNA sequence of $R$. borealis from New Delhi, India, was deposited on the NCBI database, MT775429 (95\% identity with R. macrosoma KT003748 from Spain and 94\% identity with R. borealis MK558206 from Belgium). All these concerns prompted us to carry out an integrative taxonomic analysis of $R$. borealis from the Netherlands in order to confirm the validity of these species or their synonymization with R. macrosoma.

The objectives of this study were (1) to morphometrically and molecularly characterize several populations of $R$. macrosoma from Europe and a population of $R$. borealis from the Netherlands, as well as paratypes of both species deposited in Nematode Collections, and to compare them with previous records; (2) to study the phylogenetic relationships of the European and Dutch populations of $R$. macrosoma and $R$. borealis and compare them with available sequenced populations of these species to establish their validity; and (3) to provide a clear view of the global distribution and the current climatic conditions that affect the distribution of species within the genus Rotylenchulus.

\section{Results}

2.1. Morphometric Comparison of Paratypes and Several Populations of Rotylenchulus Borealis and Rotylenchulus Macrosoma

We detected similar morphological traits in the comparison of 12 populations of $R$. borealis and 16 populations of $R$. macrosoma (Figure 1, Tables $1-8$ ), but ordinary morphometric differences among both species grouped within the three main diagnostic characters of immature females originally used for separating both species (namely, body length, stylet length, and hyaline tail region length) (Figure 2), being the major differences in the original species descriptions. Our data indicated that mean body length of all 12 populations of $R$. borealis was $401.7 \mu \mathrm{m}$, whereas the mean for 16 populations of $R$. macrosoma was $483.0 \mu \mathrm{m}$. Similarly, stylet and hyaline tail region lengths were $14.25 \mu \mathrm{m}, 7.8 \mu \mathrm{m}$ vs. $17.28 \mu \mathrm{m}, 12.5 \mu \mathrm{m}$, respectively (Tables 2-8). No differences were detected between the paratype immature females and males of $R$. borealis and the original description, as well as the new studied population from Huissen, Betuwe region (close to the type locality), the Netherlands (Table 2). However, of the two paratype immature females of R. macrosoma examined from Wageningen Nematode Collection (WANECO) and United States Department of Agriculture (USDA) nematode collections, both specimens showed a stylet length slightly lower than $18.0 \mu \mathrm{m}$ (Table 5), and representing a lower measure to that provided in the type population from olive at Hulda, Israel, and quite close to several European populations, such as Spanish populations from Jerez and Huévar del Aljarafe, Cretan populations from Petrokefali and Limnes, or the Rwandan population from Burera. Nevertheless, immature female body and hyaline tail region lengths were similar to those provided in the original description. 


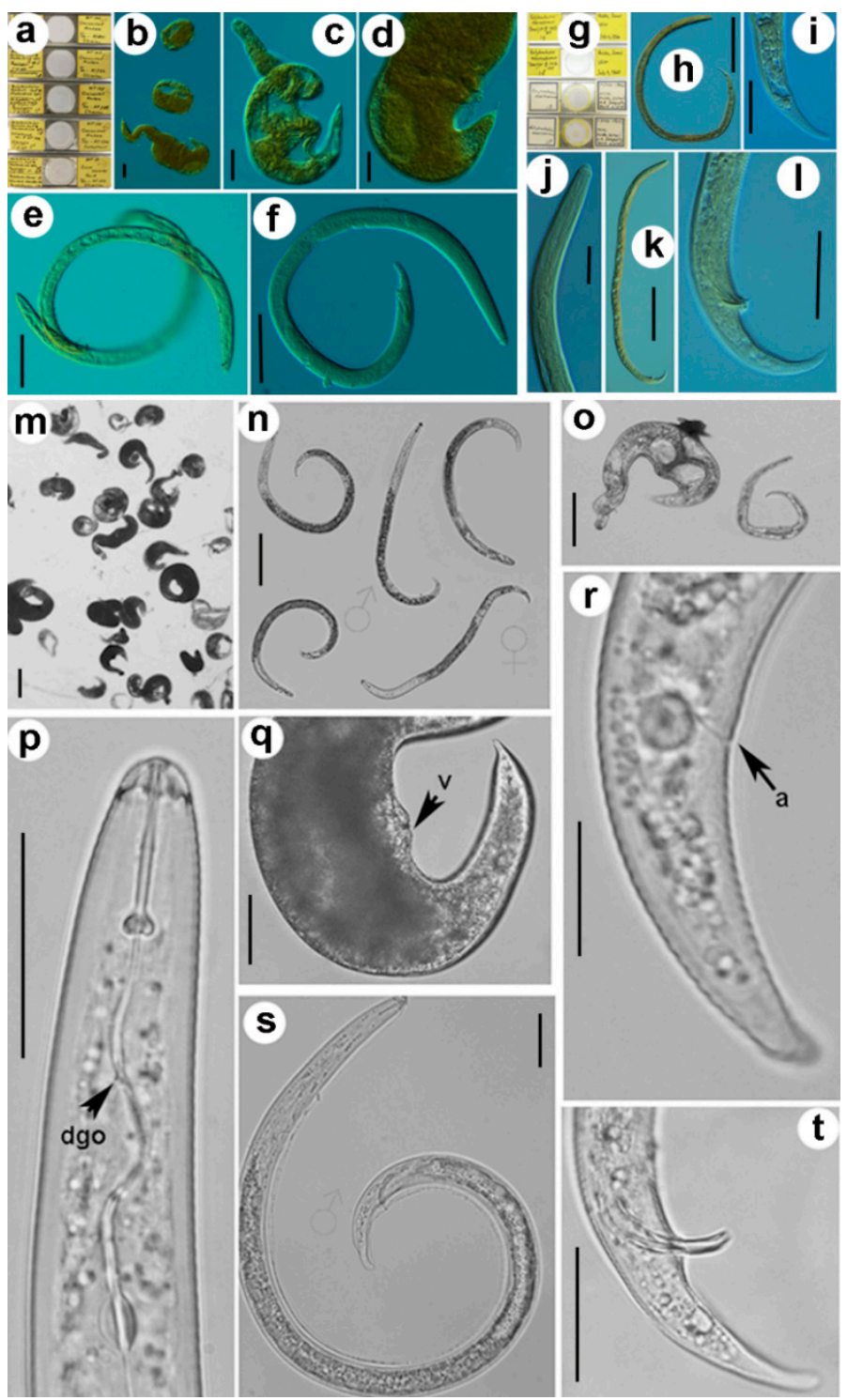

Figure 1. Comparative morphology among paratype specimens of Rotylenchulus borealis from the Netherlands (a-f), paratype specimens of Rotylenchulus macrosoma from Israel (g-l), and a population of Rotylenchulus macrosoma from Hungary $(\mathbf{m}-\mathbf{t})$. (a,g) slides deposited in Wageningen Nematode Collection (WANECO) and United States Department of Agriculture (USDA) nematode collections; (b-d,m,o, $\mathbf{q})$ mature females; $(\mathbf{e}, \mathbf{h}-\mathbf{j}, \mathbf{n}, \mathbf{p}, \mathbf{r})$ immature females; $(\mathbf{f}, \mathbf{k}, \mathbf{l}, \mathbf{n}, \mathbf{s}, \mathbf{t})=$ males. Abbreviations: $\mathbf{a}=$ anus; dgo = dorsal gland opening; V = vulva. Scale bars: (b-d,h,k,m-o) $100 \mu \mathrm{m} ;(\mathbf{e}, \mathbf{f}) 50 \mu \mathrm{m} ;(\mathbf{i}, \mathbf{j}, \mathbf{l}, \mathbf{p}, \mathbf{q}, \mathbf{s}, \mathbf{t}) 20 \mu \mathrm{m}$; (r) $10 \mu \mathrm{m}$. 
Table 1. Populations sampled for Rotylenchulus spp. from two localities in the Netherlands and Israel used in this study.

\begin{tabular}{ccccc}
\hline Locality, Country & Nematode Code & D2-D3 & ITS1 & coxI \\
\hline Rotylenchulus borealis & & & & \\
\hline Huissen, Betuwe region (the Netherlands) & AV23 & MW173970 & MW1742399 & MW182432 \\
Huissen, Betuwe region (the Netherlands) & AV25 & MW173971 & MW174240 & - \\
Huissen, Betuwe region (the Netherlands) & AV26 & MW173972 & - & MW182433 \\
Huissen, Betuwe region (the Netherlands) & AV27 & MW173973 & MW174241 & MW182434 \\
Huissen, Betuwe region (the Netherlands) & AV28 & MW173974 & MW174242 & MW182435 \\
Huissen, Betuwe region (the Netherlands) & AV29 & MW173975 & - & \\
Huissen, Betuwe region (the Netherlands) & AV30 & MW173976 & - & \\
\hline Rotylenchulus macrosoma & & & & - \\
\hline Beit She'an (Israel) & C26 & MW173977 & - & - \\
Beit She'an (Israel) & C27 & MW173978 & - & - \\
Beit She'an (Israel) & C29 & MW173979 & - & - \\
Beit She'an (Israel) & C30 & MW173980 & - & - \\
Beit She'an (Israel) & C31 & MW173981 & MW174243 & - \\
Beit She'an (Israel) & C32 & MW173982 & MW174244 & - \\
Beit She'an (Israel) & C45 & MW173983 & MW174245 & - \\
Beit She'an (Israel) & C46 & MW173984 & MW174246 & - \\
Beit She'an (Israel) & C47 & MW173985 & - & - \\
\hline
\end{tabular}

(-) Not obtained or not performed.

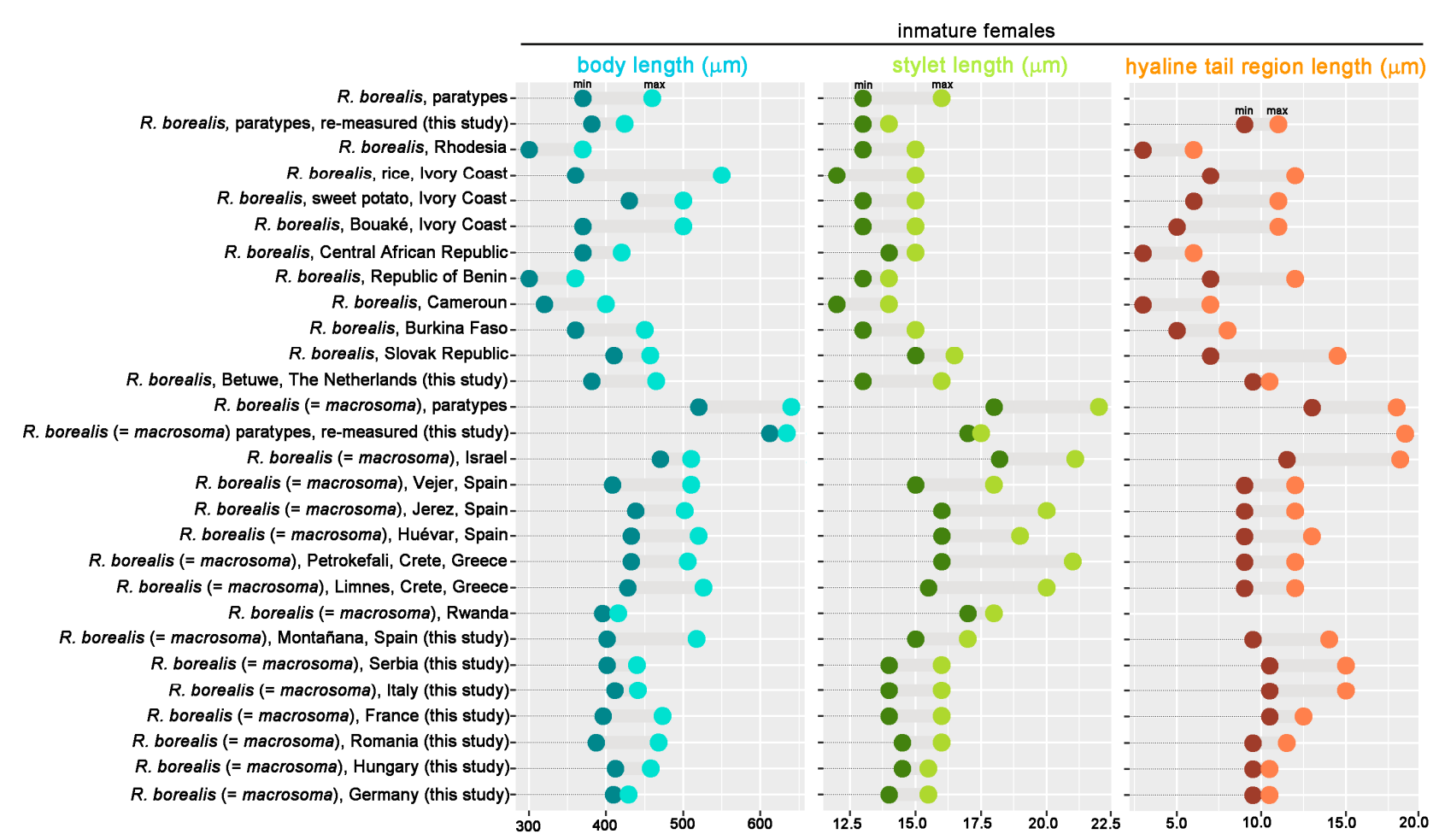

Figure 2. Range (minimum and maximum) comparative key diagnostic measures of immature females (body, stylet, and hyaline female tail lengths) for separating among $R$. borealis and R. macrosoma populations in decreasing chronological order of publication. 


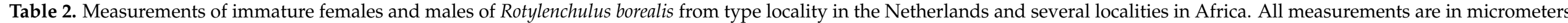
and in the form mean $\pm \mathrm{SD}$ (range).

\begin{tabular}{|c|c|c|c|c|c|c|c|c|c|c|}
\hline \multirow[b]{2}{*}{ Character/Ratio } & \multicolumn{2}{|c|}{$\begin{array}{l}\text { Grasses, Arnhem, the } \\
\text { Netherlands, Paratypes }\end{array}$} & \multicolumn{2}{|c|}{$\begin{array}{c}\text { Paratypes from WANECO } \\
\text { Nematode Collection, } \\
\text { Re-Measured in This Study }\end{array}$} & \multicolumn{2}{|c|}{$\begin{array}{c}\text { (R. variabilis }=\text { R. borealis) Rumex } \\
\text { sp., Inyanga Orchard Area, } \\
\text { Southern Rhodesia } \\
\text { (Dasgupta et al. [10] }\end{array}$} & \multicolumn{2}{|c|}{$\begin{array}{l}\text { Upland Rice, Ferkessedougou, } \\
\text { Ivory Coast [12] }\end{array}$} & \multicolumn{2}{|c|}{$\begin{array}{l}\text { Sweet Potato, Ferkessedougou, } \\
\text { Ivory Coast [12] }\end{array}$} \\
\hline & $\begin{array}{l}\text { Immature } \\
\text { Females }\end{array}$ & Males & $\begin{array}{l}\text { Immature } \\
\text { Females }\end{array}$ & Males & $\begin{array}{l}\text { Immature } \\
\text { Females }\end{array}$ & Males & $\begin{array}{l}\text { Immature } \\
\text { Females }\end{array}$ & Males & $\begin{array}{l}\text { Immature } \\
\text { Females }\end{array}$ & Males \\
\hline $\mathrm{n}$ & 20 & 40 & 3 & 5 & 22 & 21 & 18 & 17 & 5 & 8 \\
\hline $\mathrm{L}^{\mathrm{a}}$ & $(370-460)$ & $(400-490)$ & $\begin{array}{l}408 \pm 24 \\
(381-424)\end{array}$ & $\begin{array}{c}418 \pm 12 \\
(404-435)\end{array}$ & $(300-370)$ & $(340-410)$ & $\begin{array}{c}420 \\
(360-550)\end{array}$ & $\begin{array}{c}480 \\
(400-540)\end{array}$ & $\begin{array}{c}470 \\
(430-500)\end{array}$ & $\begin{array}{c}520 \\
(480-570)\end{array}$ \\
\hline a & $(22.5-32.5)$ & $(30.3-40.2)$ & $\begin{array}{l}27.9 \pm 2.3 \\
(25.4-29.9)\end{array}$ & $\begin{array}{l}24.1 \pm 2.8 \\
(21.6-28.9)\end{array}$ & $(22-26)$ & $(22-33)$ & $\begin{array}{c}27 \\
(23-31)\end{array}$ & $\begin{array}{c}29.2 \\
(25-42)\end{array}$ & $\begin{array}{c}29 \\
(26-31)\end{array}$ & $\begin{array}{c}28 \\
(24-36)\end{array}$ \\
\hline $\mathrm{b}$ & $(2.5-3.4)$ & $(3.2-4.0)$ & $\begin{array}{l}4.1 \pm 0.2 \\
(3.9-4.2)\end{array}$ & $\begin{array}{l}3.8 \pm 0.2 \\
(3.4-3.9)\end{array}$ & $(3.3-3.9)$ & $(3.1-4.1)$ & $\begin{array}{c}2.9 \\
(2.0-3.3)\end{array}$ & $\begin{array}{c}4.4 \\
(3.8-5.2)\end{array}$ & $\begin{array}{c}3.0 \\
(2.8-3.6)\end{array}$ & $\begin{array}{c}4.5 \\
(4.5-5.0)\end{array}$ \\
\hline c & $(11.3-14.8)$ & $(12.1-15.8)$ & $\begin{array}{c}14.9 \pm 0.5 \\
(14.6-15.5)\end{array}$ & $\begin{array}{l}14.7 \pm 1.0 \\
(13.6-16.1)\end{array}$ & $(13.0-16.0)$ & $(14-20)$ & $\begin{array}{c}14.7 \\
(12.5-17.3)\end{array}$ & $\begin{array}{c}16 \\
(14-18)\end{array}$ & $\begin{array}{c}14.3 \\
(12.0-16.0)\end{array}$ & $\begin{array}{c}15 \\
(11-18)\end{array}$ \\
\hline$c^{\prime}$ & - & - & $\begin{array}{l}2.8 \pm 0.2 \\
(2.6-2.9)\end{array}$ & $\begin{array}{l}2.8 \pm 0.1 \\
(2.6-2.9)\end{array}$ & $(2.6-3.2)$ & - & $\begin{array}{c}3.4 \\
(3.0-4.0)\end{array}$ & - & $\begin{array}{c}3.5 \\
(3.1-4.4)\end{array}$ & - \\
\hline $\mathrm{V}$ or $\mathrm{T}$ & $(57.6-64.8)$ & $(25.0-54.0)$ & $\begin{array}{l}62.2 \pm 1.0 \\
(61.0-63.0)\end{array}$ & $\begin{array}{l}39.7 \pm 13.5 \\
(23.4-56.4)\end{array}$ & $(59-66)$ & $(29-51)$ & $\begin{array}{c}61 \\
(57-67)\end{array}$ & - & $\begin{array}{c}62 \\
(59-66)\end{array}$ & - \\
\hline o & - & - & $\begin{array}{c}124.4 \pm 3.7 \\
(121.4-128.6)\end{array}$ & $\begin{array}{c}136.7 \pm 8.1 \\
(130.8-150.0)\end{array}$ & $(120-138)$ & - & $\begin{array}{c}131 \\
(113-160)\end{array}$ & - & $\begin{array}{c}124 \\
(113-147)\end{array}$ & - \\
\hline DGO & - & - & $\begin{array}{c}17.0 \pm 1.0 \\
(16.0-18.0)\end{array}$ & $\begin{array}{c}17.2 \pm 0.8 \\
(16.0-18.0)\end{array}$ & - & - & - & - & - & - \\
\hline Stylet length & $(13.0-16.0)$ & $(12.0-14.0)$ & $\begin{array}{c}13.7 \pm 0.6 \\
(13.0-14.0)\end{array}$ & $\begin{array}{l}12.6 \pm 0.5 \\
(12.0-13.0)\end{array}$ & $(13.0-15.0)$ & $(10.0-12.0)$ & $\begin{array}{c}14.0 \\
(12.0-15.0)\end{array}$ & $\begin{array}{c}13.0 \\
(10.0-14.0)\end{array}$ & $\begin{array}{c}14.5 \\
(13.0-15.0)\end{array}$ & $\begin{array}{c}14.0 \\
(13.0-14.0)\end{array}$ \\
\hline Lip region width & - & - & $\begin{array}{l}6.3 \pm 0.6 \\
(6.0-7.0)\end{array}$ & $\begin{array}{l}6.5 \pm 0.5 \\
(6.0-7.0)\end{array}$ & - & - & - & - & - & - \\
\hline Tail length & - & - & $\begin{array}{l}27.3 \pm 1.5 \\
(26.0-29.0)\end{array}$ & $\begin{array}{l}28.6 \pm 2.3 \\
(26.0-32.0)\end{array}$ & - & - & - & - & - & - \\
\hline $\mathrm{h}$ & - & - & $\begin{array}{l}10.0 \pm 1.0 \\
(9.0-11.0)\end{array}$ & $\begin{array}{l}9.9 \pm 0.7 \\
(9.0-11.0)\end{array}$ & $(3.0-6.0)$ & $(3.0-7.0)$ & $\begin{array}{c}9.0 \\
(7.0-12.0)\end{array}$ & $\begin{array}{c}8.0 \\
(6.0-10.0)\end{array}$ & $\begin{array}{c}8.2 \\
(6.0-11.0)\end{array}$ & $\begin{array}{c}9.0 \\
(6.0-11.0)\end{array}$ \\
\hline Spicule length & - & $(20.0-21.0)$ & - & $\begin{array}{l}21.2 \pm 0.8 \\
(20.0-22.0)\end{array}$ & - & $(19.0-23.0)$ & - & $\begin{array}{c}22.7 \\
(18.0-24.0)\end{array}$ & - & $\begin{array}{c}23.0 \\
(20.0-26.0)\end{array}$ \\
\hline Gubernaculum length & - & $(7.0-8.0)$ & - & $\begin{array}{l}7.0 \pm 0.7 \\
(6.0-8.0)\end{array}$ & - & $(7.0-9.0)$ & - & $\begin{array}{c}9.0 \\
(6.0-10.0)\end{array}$ & - & $\begin{array}{c}10.0 \\
(8.0-12.0)\end{array}$ \\
\hline
\end{tabular}

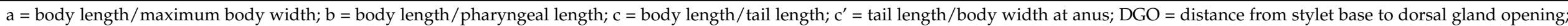

$\mathrm{h}=$ hyaline tail region length; $\mathrm{o}=(\mathrm{DGO} /$ stylet length $) \times 100 ; \mathrm{V}=$ (distance from anterior end to vulva/body length $) \times 100$ 


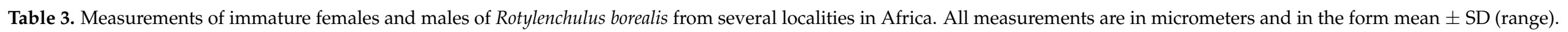

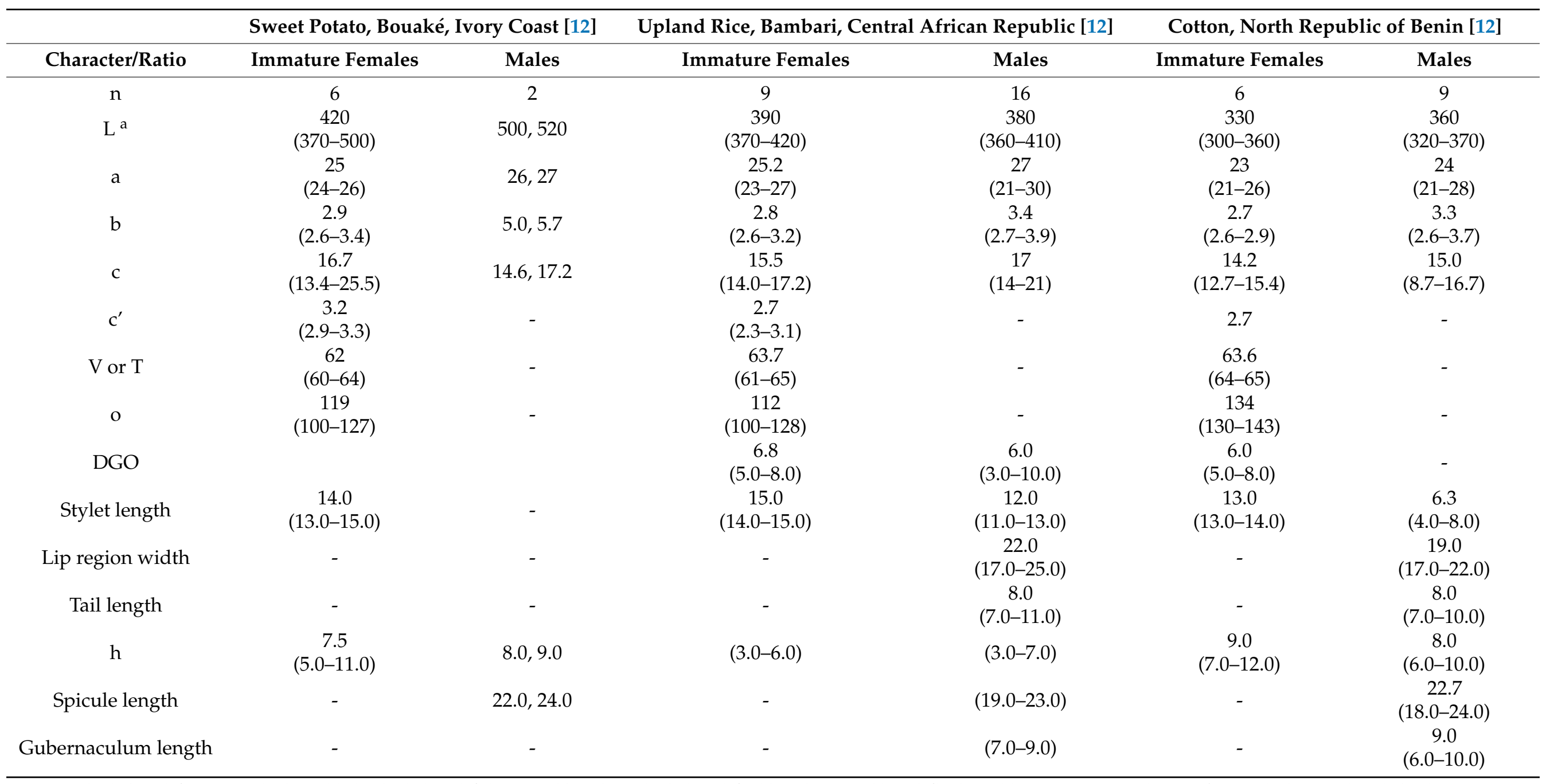

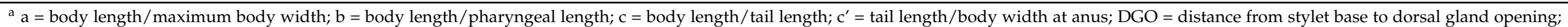

$\mathrm{h}=$ hyaline tail region length; $\mathrm{o}=(\mathrm{DGO} /$ stylet length $) \times 100 ; \mathrm{V}=$ (distance from anterior end to vulva/body length $) \times 100$. 


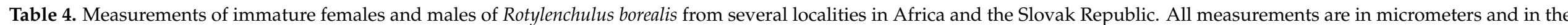
form mean $\pm \mathrm{SD}$ (range).

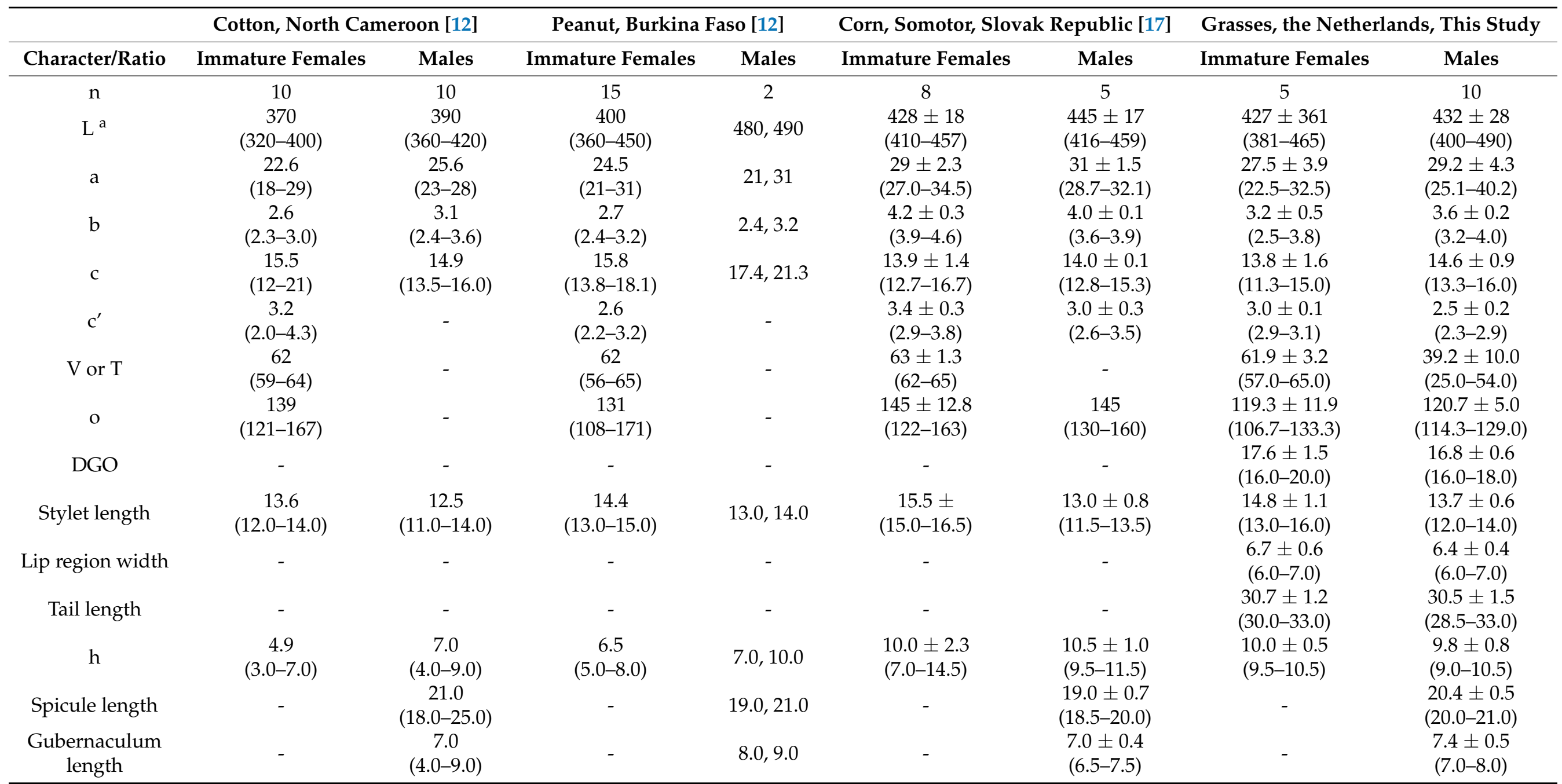

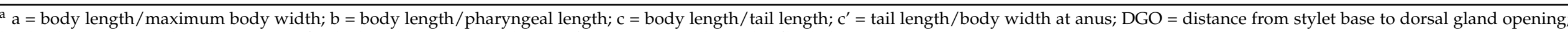
$\mathrm{h}=$ hyaline tail region length; $\mathrm{o}=(\mathrm{DGO} /$ stylet length $) \times 100 ; \mathrm{V}=($ distance from anterior end to vulva/body length $) \times 100$. 


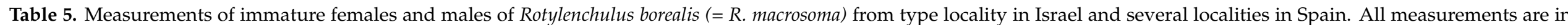
micrometers and in the form mean $\pm \mathrm{SD}$ (range).

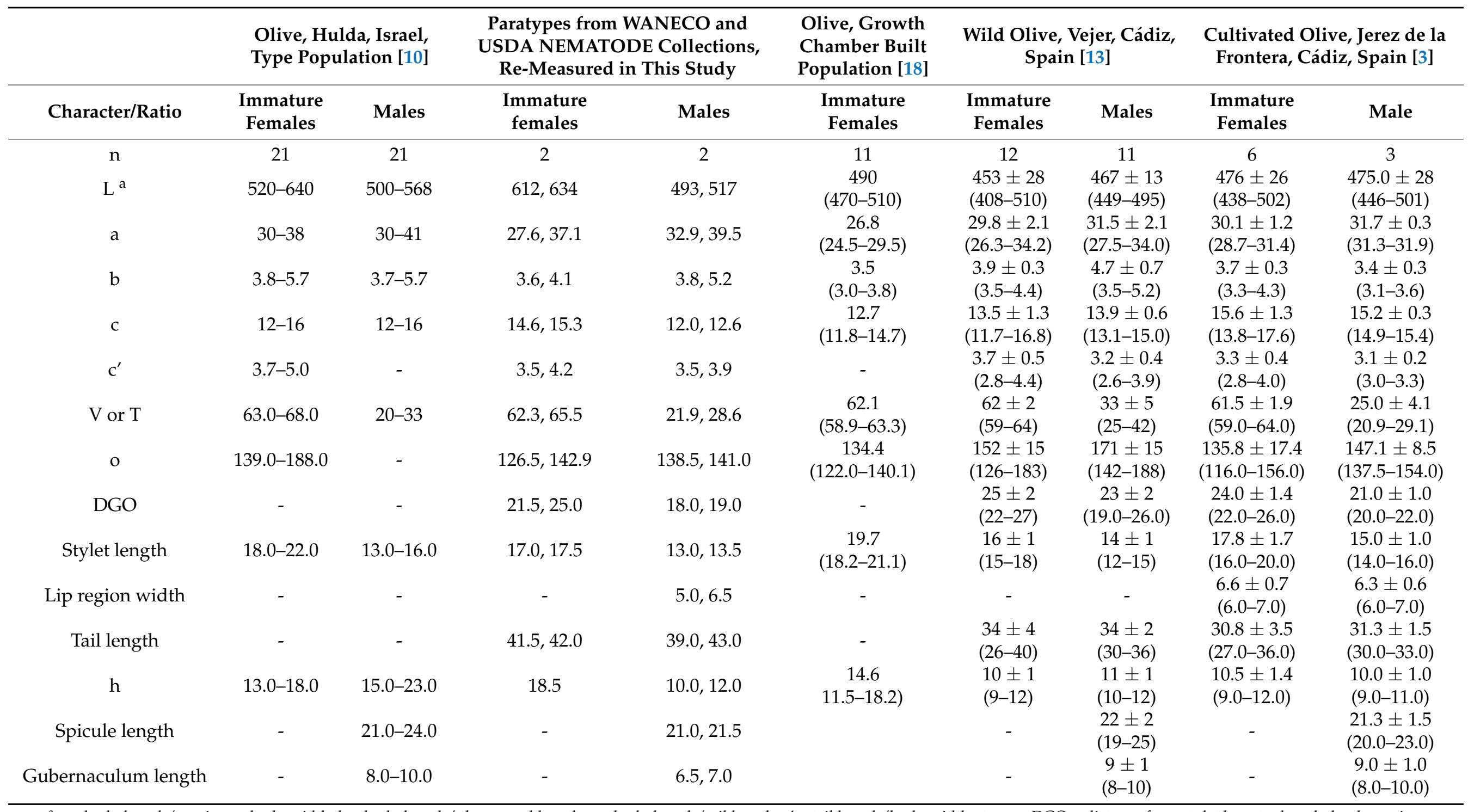

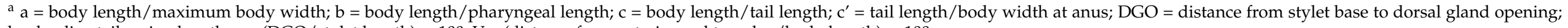




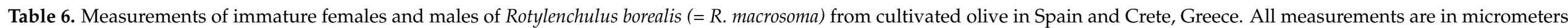
and in the form mean \pm SD (range).

\begin{tabular}{|c|c|c|c|c|c|c|}
\hline \multirow[b]{2}{*}{ Character/Ratio } & \multicolumn{2}{|c|}{$\begin{array}{c}\text { Cultivated Olive, Huévar del Aljarafe, } \\
\text { Seville Province, Spain [3] }\end{array}$} & \multicolumn{2}{|c|}{ Cultivated Olive, Petrokefali, Crete, Greece [9] } & \multicolumn{2}{|c|}{ Cultivated Olive, Limnes, Crete, Greece } \\
\hline & $\begin{array}{l}\text { Immature } \\
\text { Females }\end{array}$ & Males & $\begin{array}{l}\text { Immature } \\
\text { Females }\end{array}$ & Males & $\begin{array}{l}\text { Immature } \\
\text { Females }\end{array}$ & Male \\
\hline $\mathrm{n}$ & 10 & 10 & 10 & 5 & 10 & 10 \\
\hline \multirow{2}{*}{$\mathrm{L}^{\mathrm{a}}$} & $484 \pm 30$ & $478 \pm 31$ & $467 \pm 27$ & $468 \pm 31$ & $488 \pm 31$ & $463 \pm 33$ \\
\hline & $(432-520)$ & $(432-514)$ & $(432-506)$ & $(433-503)$ & $(428-526)$ & $(418-516)$ \\
\hline a & $29.7 \pm 1.5$ & $29.9 \pm 1.4$ & $28.9 \pm 1.9$ & $30.8 \pm 2.1$ & $29.7 \pm 1.0$ & $28.2 \pm 1.8$ \\
\hline \multirow{2}{*}{$\mathrm{b}$} & $3.7 \pm 0.3$ & $3.6 \pm 0.3$ & $3.6 \pm 0.3$ & $3.4 \pm 0.3$ & $3.7 \pm 0.2$ & $3.6 \pm 0.3$ \\
\hline & $(3.3-4.3)$ & $(3.0-4.1)$ & $(3.3-4.3)$ & $(3.1-3.6)$ & $(3.4-4.1)$ & $(3.2-4.0)$ \\
\hline \multirow[b]{2}{*}{ c } & $15.4 \pm 1.2$ & $14.8 \pm 0.7$ & $15.3 \pm 0.9$ & $14.7 \pm 0.9$ & $15.4 \pm 1.0$ & $13.8 \pm 1.3$ \\
\hline & $(14.1-17.6)$ & $(13.4-15.4)$ & $(13.8-17.0)$ & $(13.1-15.4)$ & $(14.1-17.1)$ & $(11.6-15.4)$ \\
\hline \multirow{2}{*}{$c^{\prime}$} & $3.1 \pm 0.3$ & $3.0 \pm 0.1$ & $3.3 \pm 0.3$ & $3.0 \pm 0.2$ & $3.1 \pm 0.4$ & $2.9 \pm 0.1$ \\
\hline & $(2.6-4.0)$ & $(2.8-3.1)$ & $(2.8-4.0)$ & $(2.8-3.3)$ & $(2.6-3.8)$ & $(2.8-3.1)$ \\
\hline \multirow{2}{*}{$\mathrm{V}$ or $\mathrm{T}$} & $62.6 \pm 2.2$ & $27.1 \pm 3.8$ & $61.4 \pm 2.0$ & $31.8 \pm 1.3$ & $62.4 \pm 1.9$ & $31.3 \pm 5.3$ \\
\hline & $(59.0-66.0)$ & $(21.1-32.2)$ & $(58.0-64.0)$ & $(30.0-33.0)$ & $(60.0-65.0)$ & $(21.5-37.1)$ \\
\hline \multirow{2}{*}{ DGO } & $23.8 \pm 1.3$ & $21.6 \pm 1.1$ & $23.8 \pm 1.5$ & $20.8 \pm 1.3$ & $23.6 \pm 1.3$ & $21.6 \pm 1.7$ \\
\hline & $(22.0-26.0)$ & $(20.0-23.0)$ & $(21.0-26.0)$ & $(20.0-23.0)$ & $(21.0-25.0)$ & $(19.0-24.0)$ \\
\hline \multirow{2}{*}{ Stylet length } & $17.3 \pm 1.2$ & $16.0 \pm 0.9$ & $18.5 \pm 1.7$ & $14.6 \pm 0.5$ & $17.4 \pm 1.4$ & $15.4 \pm 0.5$ \\
\hline & $(16.0-19.0)$ & $(15.0-17.0)$ & $(16.0-21.0)$ & $(14.0-15.0)$ & $(15.5-20.0)$ & $(15.0-16.0)$ \\
\hline \multirow{2}{*}{ Lip region width } & $6.6 \pm 0.6$ & $6.5 \pm 0.5$ & $6.7 \pm 0.7$ & $6.6 \pm 0.5$ & $6.7 \pm 0.8$ & $6.5 \pm 0.6$ \\
\hline & $(6.0-7.5)$ & $(6.0-7.0)$ & $(6.0-7.5)$ & $(6.0-7.0)$ & $(6.0-8.0)$ & $(6.0-7.5)$ \\
\hline \multirow{2}{*}{ Tail length } & $31.5 \pm 3.2$ & $32.4 \pm 2.3$ & $30.6 \pm 2.8$ & $31.4 \pm 0.9$ & $31.8 \pm 3.0$ & $33.6 \pm 2.4$ \\
\hline & $(27.0-37.0)$ & $(29.0-35.0)$ & $(27.0-36.0)$ & $(30.0-32.0)$ & $(28.0-37.0)$ & $(29.0-37.0)$ \\
\hline \multirow{2}{*}{$\mathrm{h}$} & $10.8 \pm 1.6$ & $10.6 \pm 0.5$ & $10.4 \pm 1.1$ & $10.2 \pm 1.3$ & $10.6 \pm 1.3$ & $10.5 \pm 0.8$ \\
\hline & $(9.0-13.0)$ & $(10.0-11.0)$ & $(9.0-12.0)$ & $(9.0-11.0)$ & $(9.0-12.0)$ & $(9.0-12.0)$ \\
\hline Spicule length & - & $22.6 \pm 1.4$ & - & $21.8 \pm 1.3$ & - & $22.4 \pm 1.4$ \\
\hline Spicule length & - & $(21.0-24.0)$ & - & $(20.0-23.0)$ & - & $(20.0-24.0)$ \\
\hline length & _- & $10.0 \pm 0.9$ & _- & $9.2 \pm 0.8$ & _ & $10.2 \pm 0.8$ \\
\hline Gubernaculum length & - & $(9.0-11.0)$ & - & $(8.0-10.0)$ & - & $(9.0-11.0)$ \\
\hline
\end{tabular}

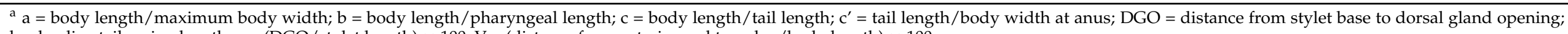

$\mathrm{h}=$ hyaline tail region length; $\mathrm{o}=(\mathrm{DGO} /$ stylet length $) \times 100 ; \mathrm{V}=($ distance from anterior end to vulva $/$ body length $) \times 100$. 


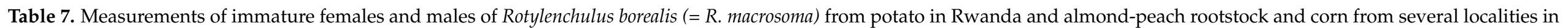
Europe. All measurements are in micrometers and in the form mean $\pm \mathrm{SD}$ (range).

\begin{tabular}{|c|c|c|c|c|c|c|c|c|}
\hline \multirow[b]{2}{*}{ Character/Ratio } & \multicolumn{2}{|c|}{ Potato, Burera, North Rwanda [14] } & \multicolumn{2}{|c|}{$\begin{array}{c}\text { Almond-Peach Rootstock, Montañana, } \\
\text { Zaragoza, Spain, This Study }\end{array}$} & \multicolumn{2}{|c|}{$\begin{array}{c}\text { Corn, Bečej, Vojvodina, Serbia, } \\
\text { This Study }\end{array}$} & \multicolumn{2}{|c|}{$\begin{array}{l}\text { Corn, Moretta, Cuneo, Italy, } \\
\text { This Study }\end{array}$} \\
\hline & $\begin{array}{l}\text { Immature } \\
\text { Females }\end{array}$ & Males & $\begin{array}{l}\text { Immature } \\
\text { Females }\end{array}$ & Males & $\begin{array}{l}\text { Immature } \\
\text { Females }\end{array}$ & Males & $\begin{array}{l}\text { Immature } \\
\text { Females }\end{array}$ & Males \\
\hline $\mathrm{n}$ & 6 & 1 & 10 & 10 & 10 & 10 & 10 & 10 \\
\hline $\mathrm{L}^{\mathrm{a}}$ & $\begin{array}{c}403 \pm 8 \\
(395-416)\end{array}$ & 462 & $\begin{array}{c}461 \pm 36 \\
(401-517)\end{array}$ & $\begin{array}{c}482 \pm 33 \\
(410-533)\end{array}$ & $\begin{array}{c}425 \pm 13 \\
(401-440)\end{array}$ & $\begin{array}{c}461 \pm 39 \\
(400-505)\end{array}$ & $\begin{array}{c}427 \pm 11 \\
(411-441)\end{array}$ & $\begin{array}{c}436.7 \pm 28 \\
(405-483)\end{array}$ \\
\hline $\mathrm{a}$ & $\begin{array}{l}24.3 \pm 0.7 \\
(23.5-25.5)\end{array}$ & 29.2 & $\begin{array}{l}27.6 \pm 2.4 \\
(25.1-31.9)\end{array}$ & $\begin{array}{l}28.1 \pm 1.2 \\
(26.5-30.4)\end{array}$ & $\begin{array}{l}26.2 \pm 1.4 \\
(24.3-28.2)\end{array}$ & $\begin{array}{l}25.5 \pm 1.6 \\
(22.1-27.3)\end{array}$ & $\begin{array}{l}25.9 \pm 1.7 \\
(23.0-28.0)\end{array}$ & $\begin{array}{l}27.6 \pm 1.8 \\
(25.3-31.2)\end{array}$ \\
\hline $\mathrm{b}$ & $\begin{array}{l}4.1 \pm 0.1 \\
(4.0-4.3)\end{array}$ & 4.1 & $\begin{array}{l}3.1 \pm 0.3 \\
(2.6-3.6)\end{array}$ & $\begin{array}{l}4.1 \pm 0.2 \\
(3.8-4.6)\end{array}$ & $\begin{array}{l}3.5 \pm 0.4 \\
(2.9-4.2)\end{array}$ & $\begin{array}{l}3.9 \pm 0.4 \\
(3.4-4.6)\end{array}$ & $\begin{array}{l}3.6 \pm 0.4 \\
(2.9-4.2)\end{array}$ & $\begin{array}{l}3.9 \pm 0.2 \\
(3.0-3.3)\end{array}$ \\
\hline c & $\begin{array}{c}13.1 \pm 0.7 \\
(12.4-14.2)\end{array}$ & 13.6 & $\begin{array}{c}12.6 \pm 1.3 \\
(11.0-14.9)\end{array}$ & $\begin{array}{c}13.7 \pm 1.7 \\
(11.0-17.6)\end{array}$ & $\begin{array}{c}12.6 \pm 0.7 \\
(12.0-14.0)\end{array}$ & $\begin{array}{c}15.3 \pm 1.4 \\
(13.7-17.9)\end{array}$ & $\begin{array}{c}12.6 \pm 0.8 \\
(11.8-14.5)\end{array}$ & $\begin{array}{c}13.2 \pm 0.9 \\
(12.3-14.6)\end{array}$ \\
\hline$c^{\prime}$ & $\begin{array}{l}2.9 \pm 0.3 \\
(2.5-3.3)\end{array}$ & 2.5 & $\begin{array}{l}3.5 \pm 0.3 \\
(2.8-4.0)\end{array}$ & $\begin{array}{l}2.9 \pm 0.3 \\
(2.4-3.6)\end{array}$ & $\begin{array}{l}3.7 \pm 0.2 \\
(3.4-3.9)\end{array}$ & $\begin{array}{l}2.7 \pm 0.2 \\
(2.4-3.0)\end{array}$ & $\begin{array}{l}3.6 \pm 0.2 \\
(3.2-3.8)\end{array}$ & $\begin{array}{l}3.1 \pm 0.1 \\
(3.0-3.3)\end{array}$ \\
\hline $\mathrm{V}$ or $\mathrm{T}$ & $\begin{array}{c}63.3 \pm 1.2 \\
(62.1-64.9)\end{array}$ & - & $\begin{array}{c}61.1 \pm 0.9 \\
(59.6-62.7)\end{array}$ & $\begin{array}{c}27.1 \pm 5.3 \\
(18.7-35.1)\end{array}$ & $\begin{array}{c}59.9 \pm 0.6 \\
(59-61)\end{array}$ & $\begin{array}{c}30.5 \pm 3 \\
(26-35)\end{array}$ & $\begin{array}{c}60.1 \pm 1.0 \\
(58.0-61.8)\end{array}$ & $\begin{array}{c}23.4 \pm 2.1 \\
(21.5-26.0)\end{array}$ \\
\hline $\mathrm{o}$ & - & - & $\begin{array}{c}127.4 \pm 9.6 \\
(112.5-141.2)\end{array}$ & $\begin{array}{c}137.4 \pm 15.4 \\
(117.9-163.0)\end{array}$ & $\begin{array}{c}120 \pm 7 \\
(113.3-135.5)\end{array}$ & $\begin{array}{l}131 \pm 10 \\
(102-133)\end{array}$ & $\begin{array}{c}118.0 \pm 7.9 \\
(106.3-129.0)\end{array}$ & $\begin{array}{c}122.7 \pm 7.9 \\
(114.3-143.0)\end{array}$ \\
\hline DGO & $\begin{array}{c}20.2 \pm 1.6 \\
(18.0-22.0)\end{array}$ & - & $\begin{array}{c}20.8 \pm 2.4 \\
(17.5-24.0)\end{array}$ & $\begin{array}{c}19.1 \pm 2.1 \\
(16.0-22.0)\end{array}$ & $\begin{array}{c}17.9 \pm 1.6 \\
(16.0-21.0)\end{array}$ & $\begin{array}{c}18.6 \pm 1.1 \\
(17.0-21.0)\end{array}$ & $\begin{array}{c}17.7 \pm 1.5 \\
(16.0-20.0)\end{array}$ & $\begin{array}{c}17.4 \pm 1.0 \\
(16.0-20.0)\end{array}$ \\
\hline Stylet length & $\begin{array}{c}17.3 \pm 0.3 \\
(17.0-18.0)\end{array}$ & 16.8 & $\begin{array}{c}16.3 \pm 0.8 \\
(15.0-17.0)\end{array}$ & $\begin{array}{c}13.9 \pm 0.3 \\
(13.5-14.5)\end{array}$ & $\begin{array}{c}14.9 \pm 0.6 \\
(14.0-16.0)\end{array}$ & $\begin{array}{c}14.2 \pm 0.3 \\
(13.5-14.5)\end{array}$ & $\begin{array}{c}15.0 \pm 0.7 \\
(14.0-16.0)\end{array}$ & $\begin{array}{c}14.2 \pm 0.4 \\
(13.5-15.0)\end{array}$ \\
\hline Lip region width & - & - & $\begin{array}{l}6.6 \pm 0.4 \\
(6.0-7.0)\end{array}$ & $\begin{array}{c}6.1 \pm 0.4 \\
(5.5-6.50)\end{array}$ & $\begin{array}{l}6.5 \pm 0.3 \\
(6.0-7.0)\end{array}$ & - & $\begin{array}{l}6.5 \pm 0.3 \\
(6.0-7.0)\end{array}$ & $\begin{array}{l}6.5 \pm 0.4 \\
(6.0-7.0)\end{array}$ \\
\hline Tail length & $\begin{array}{c}30.9 \pm 1.3 \\
(29.0-32.0)\end{array}$ & 34.0 & $\begin{array}{c}37.1 \pm 5.1 \\
(27.0-41.5)\end{array}$ & $\begin{array}{c}35.6 \pm 5.0 \\
(25.5-43.0)\end{array}$ & $\begin{array}{l}33.8 \pm 1.5 \\
(31.0-35.5)\end{array}$ & $\begin{array}{c}30.3 \pm 4.1 \\
(23.5-35.0)\end{array}$ & $\begin{array}{l}33.9 \pm 2.0 \\
(30.0-36.5)\end{array}$ & $\begin{array}{c}33.6 \pm 1.3 \\
(32.0-36.0)\end{array}$ \\
\hline $\mathrm{h}$ & - & - & $\begin{array}{l}12.3 \pm 1.6 \\
(9.5-14.0)\end{array}$ & $\begin{array}{l}11.6 \pm 2.0 \\
(7.5-14.5)\end{array}$ & $\begin{array}{c}12.2 \pm 1.4 \\
(10.5-15.0)\end{array}$ & $\begin{array}{l}8.7 \pm 1.2 \\
(7.0-10.0)\end{array}$ & $\begin{array}{c}12.5 \pm 1.7 \\
(10.5-15.0)\end{array}$ & $\begin{array}{l}8.5 \pm 0.9 \\
(7.0-10.0)\end{array}$ \\
\hline Spicule length & - & - & - & $\begin{array}{c}21.9 \pm 1.2 \\
(20.0-23.5)\end{array}$ & - & $\begin{array}{l}22.3 \pm 1.3 \\
(20.0-24.5)\end{array}$ & - & $\begin{array}{c}22.9 \pm 0.7 \\
(22.0-24.0)\end{array}$ \\
\hline $\begin{array}{c}\text { Gubernaculum } \\
\text { length }\end{array}$ & - & - & - & $\begin{array}{l}9.2 \pm 1.0 \\
(8.0-11.0)\end{array}$ & - & $\begin{array}{l}8.0 \pm 0.7 \\
(7.0-9.0)\end{array}$ & - & $\begin{array}{l}7.7 \pm 0.3 \\
(7.0-8.0)\end{array}$ \\
\hline
\end{tabular}

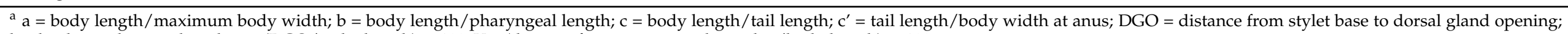

$\mathrm{h}=$ hyaline tail region length; $\mathrm{o}=(\mathrm{DGO} /$ stylet length $) \times 100 ; \mathrm{V}=$ (distance from anterior end to vulva $/$ body length $) \times 100$. 


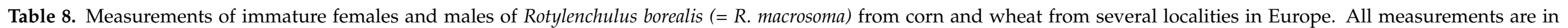
micrometers and in the form mean $\pm \mathrm{SD}$ (range).

\begin{tabular}{|c|c|c|c|c|c|c|c|c|}
\hline \multirow[b]{2}{*}{ Character/Ratio } & \multicolumn{2}{|c|}{$\begin{array}{c}\text { Corn, Le Sen, Landes, France, } \\
\text { This Study }\end{array}$} & \multicolumn{2}{|c|}{$\begin{array}{l}\text { Wheat, Mihail Kogalniceau, } \\
\text { Romania, This Study }\end{array}$} & \multicolumn{2}{|c|}{$\begin{array}{c}\text { Corn, Létavertes, Hajdú-Bihar, } \\
\text { Hungary, This Study }\end{array}$} & \multicolumn{2}{|c|}{$\begin{array}{l}\text { Corn, Möckmühl, Heilbronn, } \\
\text { Germany, This Study }\end{array}$} \\
\hline & $\begin{array}{l}\text { Immature } \\
\text { Females }\end{array}$ & Males & $\begin{array}{l}\text { Immature } \\
\text { Females }\end{array}$ & Males & $\begin{array}{l}\text { Immature } \\
\text { Females }\end{array}$ & Males & $\begin{array}{l}\text { Immature } \\
\text { Females }\end{array}$ & Males \\
\hline $\mathrm{n}$ & 10 & 10 & 10 & 10 & 10 & 10 & 10 & 10 \\
\hline $\mathrm{L}^{\mathrm{a}}$ & $\begin{array}{c}428 \pm 22 \\
(396-473)\end{array}$ & $\begin{array}{c}444 \pm 21 \\
(411-477)\end{array}$ & $\begin{array}{c}435 \pm 29 \\
(387-468)\end{array}$ & $\begin{array}{c}435 \pm 25 \\
(409-485)\end{array}$ & $\begin{array}{c}430 \pm 14 \\
(412-458)\end{array}$ & $\begin{array}{c}432 \pm 19.3 \\
(411-474)\end{array}$ & $\begin{array}{l}422 \pm 16 \\
(409-429)\end{array}$ & $\begin{array}{c}417 \pm 7 \\
(409-429)\end{array}$ \\
\hline $\mathrm{a}$ & $\begin{array}{l}26.3 \pm 1.3 \\
(24.2-28.3)\end{array}$ & $\begin{array}{l}28.3 \pm 1.8 \\
(25.7-31.2)\end{array}$ & $\begin{array}{l}27.3 \pm 1.4 \\
(24.9-29.1)\end{array}$ & $\begin{array}{l}28.1 \pm 1.8 \\
(25.6-30.3)\end{array}$ & $\begin{array}{l}27.0 \pm 0.8 \\
(25.8-28.3)\end{array}$ & $\begin{array}{l}28.9 \pm 2.5 \\
(26.3-32.2)\end{array}$ & $\begin{array}{l}27.3 \pm 0.7 \\
(26.2-28.3)\end{array}$ & $\begin{array}{l}27.6 \pm 1.6 \\
(25.6-29.8)\end{array}$ \\
\hline $\mathrm{b}$ & $\begin{array}{l}3.6 \pm 0.4 \\
(3.2-4.3)\end{array}$ & $\begin{array}{l}3.9 \pm 0.2 \\
(3.6-4.1)\end{array}$ & $\begin{array}{l}3.7 \pm 0.3 \\
(3.3-4.2)\end{array}$ & $\begin{array}{l}3.8 \pm 0.2 \\
(3.5-4.0)\end{array}$ & $\begin{array}{l}3.7 \pm 0.1 \\
(3.6-4.0)\end{array}$ & $\begin{array}{l}3.8 \pm 0.1 \\
(3.5-4.0)\end{array}$ & $\begin{array}{l}3.6 \pm 0.1 \\
(3.4-3.8)\end{array}$ & $\begin{array}{l}3.7 \pm 0.2 \\
(3.4-4.1)\end{array}$ \\
\hline C & $\begin{array}{c}12.8 \pm 0.8 \\
(11.4-14.3)\end{array}$ & $\begin{array}{c}13.5 \pm 0.5 \\
(12.6-14.0)\end{array}$ & $\begin{array}{c}14.0 \pm 0.9 \\
(12.6-15.5)\end{array}$ & $\begin{array}{c}13.0 \pm 0.5 \\
(12.6-14.0)\end{array}$ & $\begin{array}{c}14.1 \pm 0.4 \\
(13.5-14.8)\end{array}$ & $\begin{array}{c}14.1 \pm 1.0 \\
(12.4-15.4)\end{array}$ & $\begin{array}{c}14.0 \pm 0.2 \\
(13.7-14.3)\end{array}$ & $\begin{array}{c}13.1 \pm 1.0 \\
(11.6-15.3)\end{array}$ \\
\hline$c^{\prime}$ & $\begin{array}{l}3.2 \pm 0.2 \\
(2.9-3.4)\end{array}$ & $\begin{array}{l}3.0 \pm 0.2 \\
(2.7-3.2)\end{array}$ & $\begin{array}{l}3.0 \pm 0.2 \\
(2.8-3.3)\end{array}$ & $\begin{array}{l}3.1 \pm 0.2 \\
(2.8-3.3)\end{array}$ & $\begin{array}{l}3.0 \pm 0.1 \\
(2.9-3.3)\end{array}$ & $\begin{array}{l}2.9 \pm 0.2 \\
(2.7-3.3)\end{array}$ & $\begin{array}{l}3.0 \pm 0.1 \\
(2.9-3.3)\end{array}$ & $\begin{array}{l}3.1 \pm 0.2 \\
(2.8-3.3)\end{array}$ \\
\hline $\mathrm{V}$ or $\mathrm{T}$ & $\begin{array}{c}60.5 \pm 0.8 \\
(59.5-62.0)\end{array}$ & $\begin{array}{c}31.0 \pm 6.8 \\
(26.5-43.0)\end{array}$ & $\begin{array}{c}60.3 \pm 0.9 \\
(58.1-61.5)\end{array}$ & $\begin{array}{c}32.2 \pm 3.1 \\
(29.5-37.1)\end{array}$ & $\begin{array}{c}60.6 \pm 0.5 \\
(60.0-61.5)\end{array}$ & $\begin{array}{c}33.9 \pm 7.4 \\
(27.0-48.1)\end{array}$ & $\begin{array}{c}60.6 \pm 0.5 \\
(60.0-61.5)\end{array}$ & $\begin{array}{c}33.9 \pm 7.4 \\
(27.0-48.1)\end{array}$ \\
\hline DGO & $\begin{array}{c}17.9 \pm 1.5 \\
(16.0-20.0)\end{array}$ & $\begin{array}{l}16.8 \pm 0.91 \\
(15.0-18.0)\end{array}$ & $\begin{array}{c}19.0 \pm 1.9 \\
(16.0-21.0)\end{array}$ & $\begin{array}{c}17.5 \pm 0.6 \\
(17.0-18.5)\end{array}$ & $\begin{array}{c}18.3 \pm 1.3 \\
(16.0-20.0)\end{array}$ & $\begin{array}{c}17.3 \pm 1.0 \\
(15.0-18.5)\end{array}$ & $\begin{array}{c}18.6 \pm 1.1 \\
(17.0-20.0)\end{array}$ & $\begin{array}{c}17.0 \pm 0.8 \\
(15.0-18.0)\end{array}$ \\
\hline Stylet length & $\begin{array}{c}14.9 \pm 0.6 \\
(14.0-16.0)\end{array}$ & $\begin{array}{c}14.3 \pm 0.9 \\
(14.0-15.0)\end{array}$ & $\begin{array}{c}15.3 \pm 0.5 \\
(14.5-16.0)\end{array}$ & $\begin{array}{c}14.1 \pm 0.6 \\
(13.0-15.0)\end{array}$ & $\begin{array}{c}15.1 \pm 0.4 \\
(14.5-15.5)\end{array}$ & $\begin{array}{c}13.8 \pm 0.5 \\
(13.0-14.5)\end{array}$ & $\begin{array}{c}14.9 \pm 0.6 \\
(14.0-15.5)\end{array}$ & $\begin{array}{c}14.0 \pm 0.4 \\
(13.5-15.0)\end{array}$ \\
\hline Lip region width & $\begin{array}{l}6.6 \pm 0.3 \\
(6.0-7.0)\end{array}$ & $\begin{array}{l}6.5 \pm 0.3 \\
(6.0-7.0)\end{array}$ & $\begin{array}{l}6.6 \pm 0.2 \\
(6.5-7.0)\end{array}$ & $\begin{array}{l}6.5 \pm 0.4 \\
(6.0-7.0)\end{array}$ & $\begin{array}{l}6.7 \pm 0.3 \\
(6.5-7.0)\end{array}$ & $\begin{array}{l}6.6 \pm 0.4 \\
(6.0-7.0)\end{array}$ & $\begin{array}{l}6.6 \pm 0.3 \\
(6.0-7.0)\end{array}$ & $\begin{array}{l}6.4 \pm 0.5 \\
(6.0-7.0)\end{array}$ \\
\hline Tail length & $\begin{array}{c}33.5 \pm 1.4 \\
(31.0-35.5)\end{array}$ & $\begin{array}{c}33.3 \pm 0.7 \\
(32.0-34.0)\end{array}$ & $\begin{array}{c}31.0 \pm 1.5 \\
(29.0-34.0)\end{array}$ & $\begin{array}{c}31.8 \pm 1.9 \\
(32.0-38.0)\end{array}$ & $\begin{array}{c}30.6 \pm 1.3 \\
(29.0-33.0)\end{array}$ & $\begin{array}{c}31.2 \pm 2.3 \\
(28.0-36.0)\end{array}$ & $\begin{array}{l}30.1 \pm 1.0 \\
(28.0-31.0)\end{array}$ & $\begin{array}{l}32.5 \pm 2.0 \\
(28.0-36.0)\end{array}$ \\
\hline $\mathrm{h}$ & $\begin{array}{c}11.4 \pm 0.8 \\
(10.5-12.5)\end{array}$ & $\begin{array}{l}10.0 \pm 1.5 \\
(8.0-15.5)\end{array}$ & $\begin{array}{l}10.4 \pm 0.6 \\
(9.5-11.5)\end{array}$ & $\begin{array}{l}10.3 \pm 1.8 \\
(8.0-12.5)\end{array}$ & $\begin{array}{l}10.1 \pm 0.4 \\
(9.5-10.5)\end{array}$ & $\begin{array}{l}9.9 \pm 0.6 \\
(9.0-11.0)\end{array}$ & $\begin{array}{l}10.0 \pm 0.4 \\
(9.5-10.5)\end{array}$ & $\begin{array}{l}9.7 \pm 1.3 \\
(8.0-12.5)\end{array}$ \\
\hline Spicule length & - & $\begin{array}{c}22.5 \pm 0.6 \\
(22.0-23.5)\end{array}$ & - & $\begin{array}{c}22.2 \pm 0.3 \\
(22.0-23.0)\end{array}$ & - & $\begin{array}{c}21.8 \pm 2.5 \\
(21.0-22.5)\end{array}$ & - & $\begin{array}{l}21.6 \pm 0.8 \\
(20.0-22.5)\end{array}$ \\
\hline Gubernaculum length & - & $\begin{array}{l}7.8 \pm 0.3 \\
(7.5-8.0)\end{array}$ & - & $\begin{array}{l}7.7 \pm 0.4 \\
(7.0-8.0)\end{array}$ & - & $\begin{array}{l}7.5 \pm 0.4 \\
(7.0-8.0)\end{array}$ & - & $\begin{array}{l}7.4 \pm 0.4 \\
(7.0-8.0)\end{array}$ \\
\hline
\end{tabular}

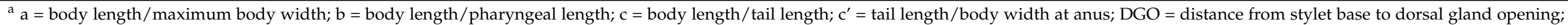

$\mathrm{h}=$ hyaline tail region length; $\mathrm{o}=(\mathrm{DGO} /$ stylet length $) \times 100 ; \mathrm{V}=$ (distance from anterior end to vulva $/$ body length $) \times 100$. 
2.2. Molecular Characterisation and Phylogenetic Analysis of Rotylenchulus Borealis and Rotylenchulus Macrosoma Populations

The amplification of D2-D3 expansion domains of $28 S$ rRNA, ITS1 rRNA, and coxI genes of $R$. borealis and $R$. macrosoma populations yielded single fragments of $\approx 900 \mathrm{bp}$, $1100 \mathrm{bp}$, and $450 \mathrm{bp}$, respectively, on the basis of gel electrophoresis and, in the case of the Israel population, from cloning of the PCR product. Sixteen new sequences from the D2-D3 of 28S rRNA gene and eight new sequences from ITS1 rRNA gene were obtained in this study (7 and 9, and 4 and 4 , from the Netherlands and Israel, respectively). Four new coxI sequences from the Netherlands were deposited in GenBank; however, due to lack of material, it was not possible to obtain coxI sequences from Israel. Type B-D2D3 sequence of $R$. macrosoma from Israel was obtained for the first time in this study (MW173975). D2-D3 for R. borealis (MW173970-MW173976) showed a low intraspecific variability with $1-5$ different nucleotides and 0 indels ( $99 \%$ similarity). Similarly, intraspecific variability for D2-D3 in R. macrosoma from Israel was slightly higher, with 6-17 different nucleotides and 0-2 indels (97-99\% similarity). The molecular diversity of this marker between $R$. borealis (MW173970-MW173976) from the Netherlands and R. macrosoma (MW173977MW173985) from Israel populations was also low, with 5-22 different nucleotides and 0-2 indels (96-99\% similarity). D2-D3 sequences of R. macrosoma from Israel (MW173977MW173985) differed in 0-10 nucleotides and 0 indels (99\% similarity) when compared with sequences of R. macrosoma deposited in the NCBI database from Spain, Belgium, Serbia, Romania, Hungary, and Portugal, and with Rotylenchulus sp. 191_7 (MK558208) and R. borealis (MT775429) from Ethiopia and New Delhi in 32, 44 bp, 0, 1 indels (95\%, 94\% similarity), respectively. Similarly, D2-D3 sequences of $R$. borealis from the Netherlands (MW173970-MW173976) differed in 14-21 nucleotides and 0 indels (97-98\% similarity) when compared with sequences of $R$. macrosoma deposited in the NCBI database from Spain, Belgium, Serbia, Romania, Hungary, and Portugal, and with Rotylenchulus sp. 191_7 (MK558208) and R. borealis (MT775429) from Ethiopia and New Delhi in 41, 39 bp, 0 indels (94\%, 94\% similarity), respectively.

The ITS1 region showed a low intraspecific variability for $R$. borealis (MW174239MW174242) from the Netherlands, with 0-6 different nucleotides and 0-1 indels (98-100\% similarity). Similarly, intraspecific variability for ITS1 in R. macrosoma from Israel (MW174243MW174246) was low, with 0-11 different nucleotides and 0-4 indels (98-100\% similarity). The molecular diversity of this marker between R. borealis from the Netherlands (MW174239MW174242) and R. macrosoma from Israel (MW174243-MW174246) populations was also low, with 0-24 different nucleotides and 0-12 indels (95-100\% similarity). ITS1 sequences of $R$. macrosoma from Israel (MW174243-MW174246) differed in 19-32 nucleotides and 1-8 indels (94-96\% similarity) when compared with sequences of $R$. macrosoma deposited in the NCBI database from Spain and Greece, and with Rotylenchulus reniformis (KF999979) from Japan in 92 bp, 26 indels ( $86 \%$ similarity). Similarly, ITS1 sequences of R. borealis from the Netherlands (MW174239-MW174242) differed in 13-42 nucleotides and 1-11 indels (94-98\% similarity) when compared with sequences of $R$. macrosoma deposited in the NCBI database from Spain and Greece, and with $R$. reniformis (KP018567) from China in 137 bp, 54 indels (83\% similarity).

The partial coxI gene for $R$. borealis from the Netherlands (MW182432-MW182435) showed a low intraspecific variability with $0-8$ different nucleotides and 0 indels (98-100\% similarity). These sequences differed in 0-47 nucleotides and 0 indels (89-100\% similarity) with sequences of R. macrosoma deposited in the NCBI database from Spain, Serbia, Romania, Hungary, and Greece, and with Rotylenchulus parvus (MK558211) from Tanzania in $64 \mathrm{bp}, 4$ indels ( $85 \%$ similarity). All molecular markers suggest that populations of $R$. borealis from the Netherlands and R. macrosoma from Israel are conspecific.

Phylogenetic relationships among Rotylenchulus species inferred from analyses of D2-D3 expansion domains of $28 \mathrm{~S}$ rRNA, ITS1, and partial coxI gene sequences using Bayesian inference (BI) are shown in Figures 3-5, respectively. The phylogenetic trees 
generated with the two nuclear and the mitochondrial markers included 123, 77, and 38 sequences, with 704, 888, and 355 positions in length, respectively (Figures 3-5). D2-D3 tree of Rotylenchulus spp. showed two moderately supported clades including R. borealis type A and type B sequences (posterior probabilities $(\mathrm{PP})=0.87,0.93$, respectively), including R. reniformis, Rotylenchulus macrodoratus, and Rotylenchulus macrosomoides (Figure 3). All sequences of $R$. borealis from the Netherlands (MW173970-MW173976) and Belgium (MK558206), as well as those of $R$. borealis (= R. macrosoma) from Israel and all the sequences from Spain, Serbia, Romania, Hungary, and Greece deposited in the NCBI database clustered together in a highly supported clade $(\mathrm{PP}=1.00)$ and were well separated $(\mathrm{PP}=1.00)$ from $28 S$ of $R$. borealis (MT775429) from New Delhi (Figure 3).

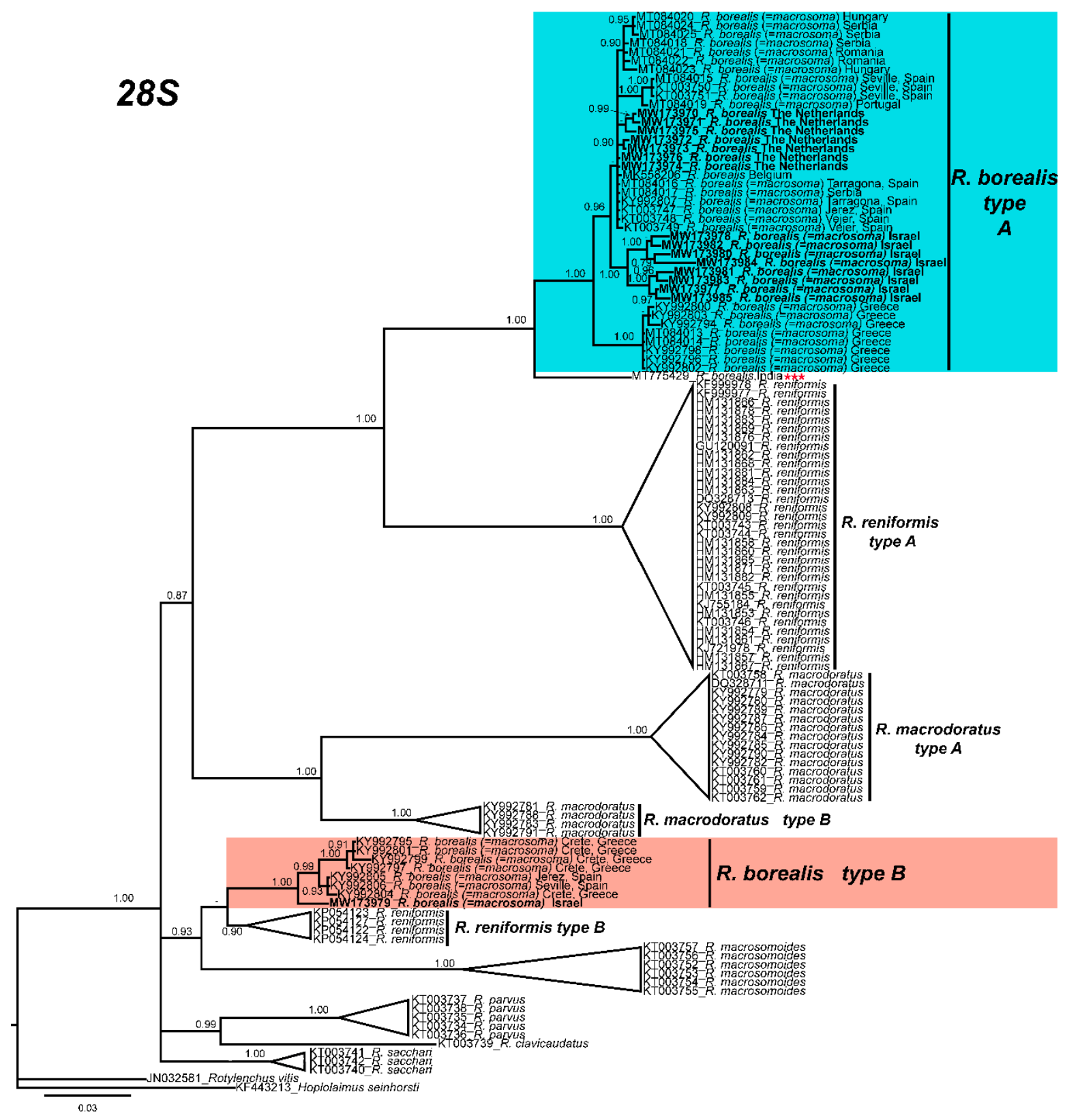

Figure 3. Phylogenetic relationships within the genus Rotylenchulus. Bayesian $50 \%$ majority rule consensus tree as inferred from D2 and D3 expansion domains of 28S rRNA sequence alignment under the general time-reversible model of sequence evolution with correction for invariable sites and a gamma-shaped distribution (GTR + I + G). Posterior probabilities of more than 0.70 are given for appropriate clades. Newly obtained sequences in this study are shown in bold. Scale bar $=$ expected changes per site. Some branches were collapsed for improving readability of Rotylenchulus species. ${ }^{* * *}$ Sequence that needs to be revised under integrative taxonomical approaches. 


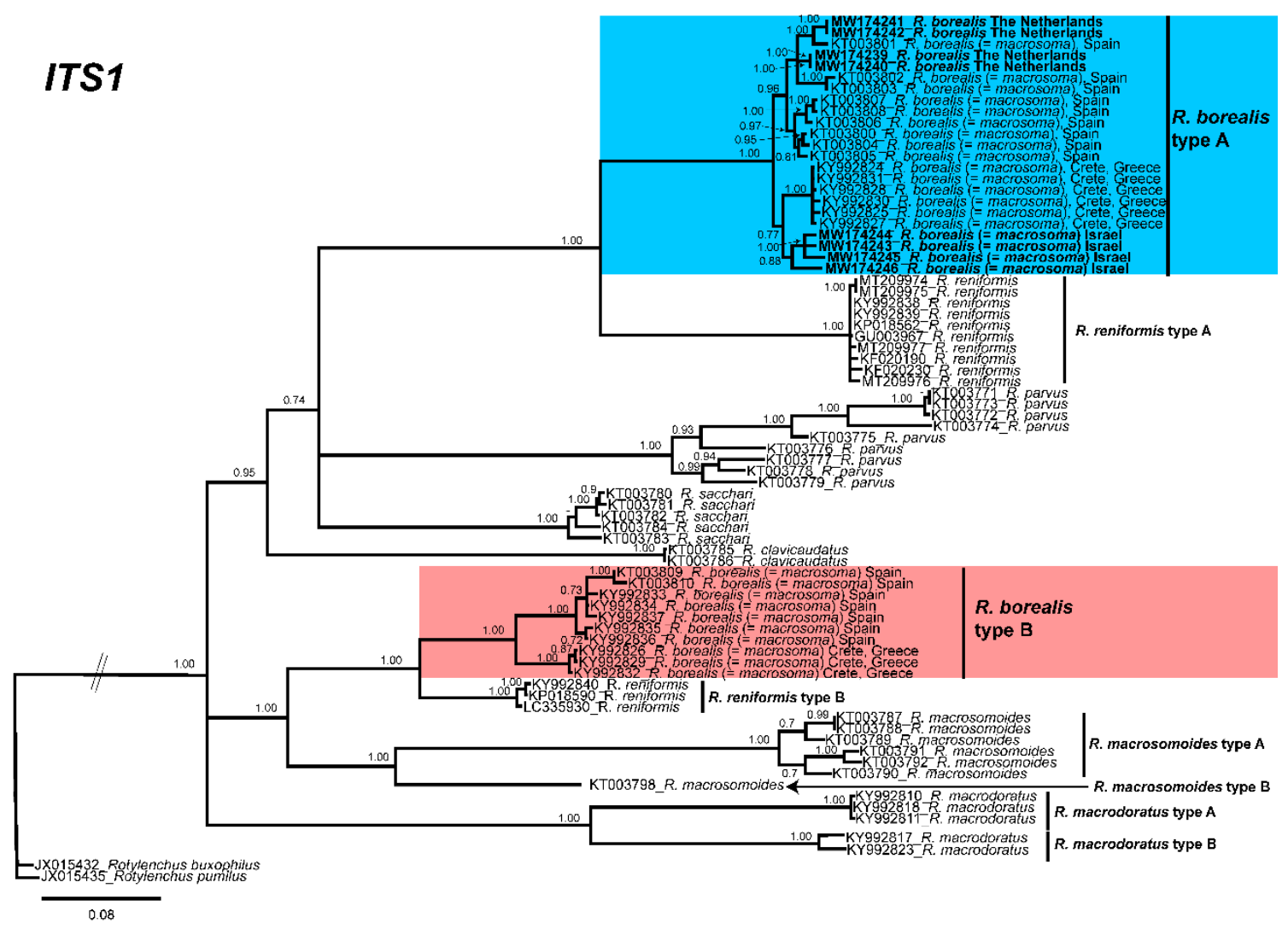

Figure 4. Phylogenetic relationships within the genus Rotylenchulus. Bayesian 50\% majority rule consensus tree as inferred from ITS1 rRNA sequence alignment under the general time-reversible model of sequence evolution with correction for invariable sites and a gamma-shaped distribution (GTR + I + G). Posterior probabilities of more than 0.70 are given for appropriate clades. Newly obtained sequences in this study are shown in bold. Scale bar = expected changes per site.

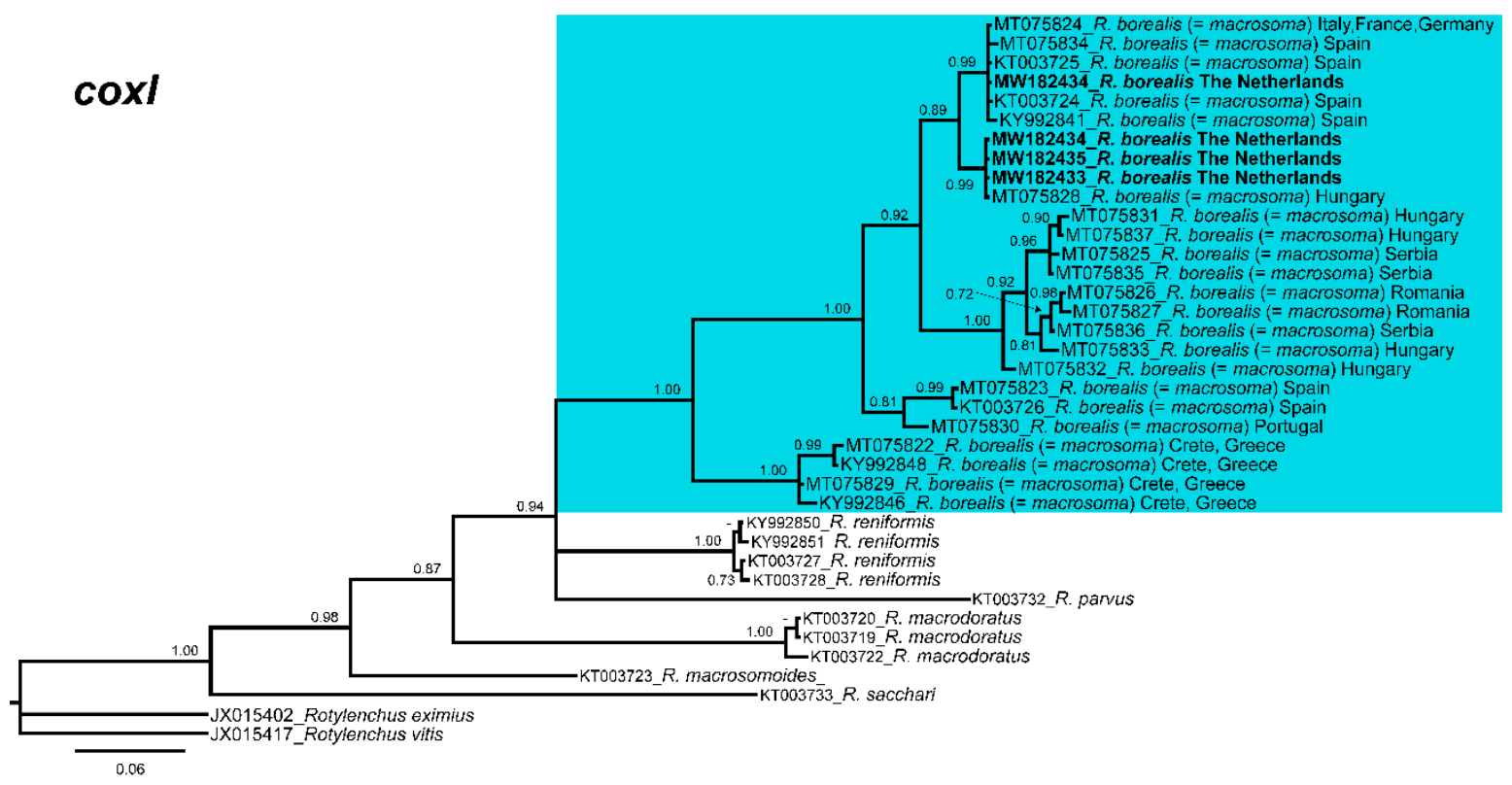

Figure 5. Phylogenetic relationships within the genus Rotylenchulus. Bayesian 50\% majority rule consensus tree as inferred from coxI mitochondrial DNA (mtDNA) sequence alignment under the general time-reversible model of sequence evolution with correction for invariable sites and a gamma-shaped distribution (GTR + I + G). Posterior probabilities of more than 0.70 are given for appropriate clades. Newly obtained sequences in this study are shown in bold. Scale bar = expected changes per site. 
The 50\% majority rule consensus ITS1 BI tree also showed two clades, one moderately and the other well supported including $R$. borealis type $\mathrm{A}$ and type B sequences $(\mathrm{PP}=0.95,1.00$, respectively), including $R$. reniformis, $R$. parvus, Rotylenchulus sacchari, and Rotylenchulus clavicaudatus (Figure 4). All sequences of $R$. borealis from the Netherlands (MW174239-MW174242) and those of R. borealis (= macrosoma) from Israel and all the sequences from Spain and Greece deposited in the NCBI database clustered together in a highly supported clade (PP = 1.00).

Finally, the phylogenetic relationships of Rotylenchulus species inferred from analysis of partial coxI gene sequences showed several clades that were well defined (Figure 5). All sequences of $R$. borealis from the Netherlands (MW182432-MW182435) and sequences from several European countries (Germany, Greece, Hungary, Italy, Portugal, Romania, Serbia, and Spain) deposited in the NCBI database clustered together in a highly supported clade $(\mathrm{PP}=1.00)$.

\subsection{Global Distribution Rotylenchulus spp.}

We detected that the genus Rotylenchulus exhibited a worldwide distribution across a wide variety of environments and climatic zones (Figure 6). We found that Rotylenchulus spp. are widely distributed in warm temperature $\left(-3^{\circ} \mathrm{C}<\right.$ annual mean temperature $<$ $+18^{\circ} \mathrm{C}$ ) and arid (annual precipitation $<300 \mathrm{~mm}$ ) climate zones, with seven different species for both types, and to a lesser extent in equatorial (annual mean temperature $\geq+18{ }^{\circ} \mathrm{C}$ ) and snow (mean temperature of the coldest month $\leq-3{ }^{\circ} \mathrm{C}$ ) climate zones, with four and one species, respectively (Figure 6). We did not detect species in the polar (mean temperature of the warmest month $<+10{ }^{\circ} \mathrm{C}$ ) climate zone (Figure 6 ). It should be noted that highest diversity of species, although less frequently found, seems to be in the southern part of Africa with mainly warm temperate and arid climatic zones (Figure 6). The species distribution observed in this study revealed that the genus Rotylenchulus is adapted to heterogeneous climatic conditions, with an annual mean temperature of $19.14{ }^{\circ} \mathrm{C}$, but ranging from 8.36 to $28.58^{\circ} \mathrm{C}$, and a mean annual precipitation of $1026.97 \mathrm{~mm}$, but ranging from 1 to $3583.00 \mathrm{~mm}$. This suggests that the occurrence of Rotylenchulus species in areas with extremely low values in annual precipitation (i.e., desert lands in Egypt and Iraq; Figure 6) could be due to the establishment of an irrigation regime in agricultural ecosystems. Only four species were reported more than three times in literature review, i.e., R. borealis (= R. macrosoma), R. macrodoratus, R. parous, and R. reniformis (Figure 6). The most widely distributed species was $R$. reniformis, followed by $R$. parvus, both reported in all continents except Antarctica (Africa, North and South America, Asia, Australia, and Europe), and R. borealis in Africa, Europe, and Middle East Asia (Figure 6). Bioclimatic variables (BIOCLIM) based on temperature (annual mean temperature (BIO1), maximum temperature of warmest month (BIO5), and minimum temperature of coldest month (BIO6)) showed significantly different temperature conditions on the distribution of these most common species (Figure 7). The two major pathogenic species (R. reniformis and R. parvus) were mainly distributed in tropical, temperate, and arid climates, showing their close relationship with warmer areas with high annual mean temperature, max temperature of the warmest month, and minimum temperature of the coldest month, ranging from 9.55 to $21.11{ }^{\circ} \mathrm{C}, 24.00$ to $3583.00 \mathrm{~mm}$ and 14.79 to $26.99{ }^{\circ} \mathrm{C}, 1.00$ to $1773.00 \mathrm{~mm}$, respectively (Figures 6 and 7). Rotylenchulus macrodoratus showed a distribution in temperate climate with annual mean temperature and precipitation ranging from 12.32 to $19.23{ }^{\circ} \mathrm{C}$ and 526.00 to $1013.00 \mathrm{~mm}$, respectively (Figure 7). The climatic plasticity of $R$. borealis is remarkable in relationship with annual mean temperature and precipitation, ranging from 8.36 to $28.58{ }^{\circ} \mathrm{C}$ and 160.00 to $1998.00 \mathrm{~mm}$, respectively (Figure 7). Rotylenchulus borealis $(=$ R. macrosoma $)$ showed statistically significant differences in lower annual mean temperature, max temperature of the warmest month, and min temperature of the coldest month in comparison to $R$. parvus and R. reniformis (Figure 7). However, only $R$. reniformis showed statistically significant differences in higher annual precipitation in comparison to the other studied species (Figure 7). Other bioclimatic variables are shown in Figure S1. 


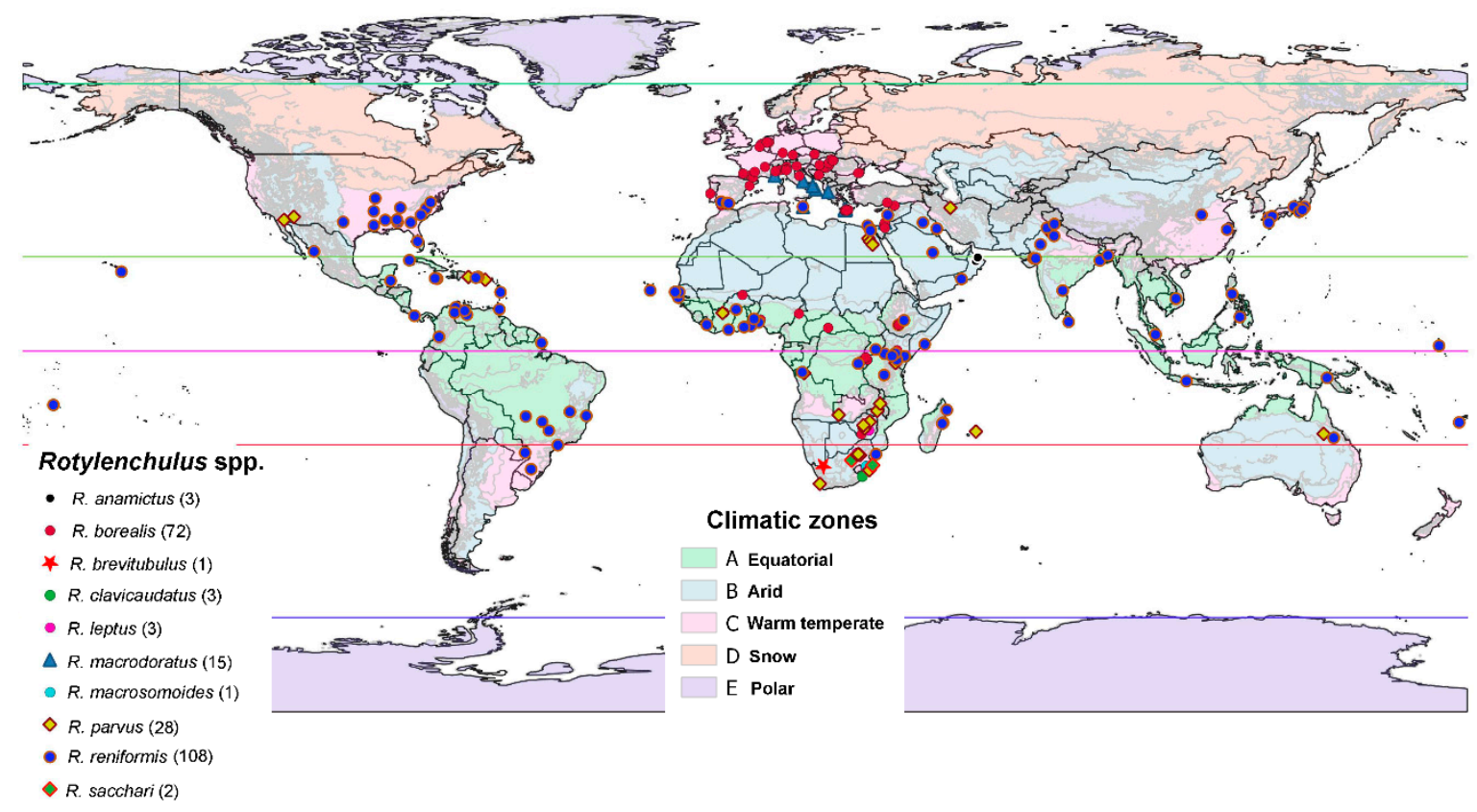

Figure 6. World map distribution of Rotylenchulus species across different climate conditions. Climatic zones based on type of vegetation [19]. In the species list, the number in brackets indicates the locations cited for each species.
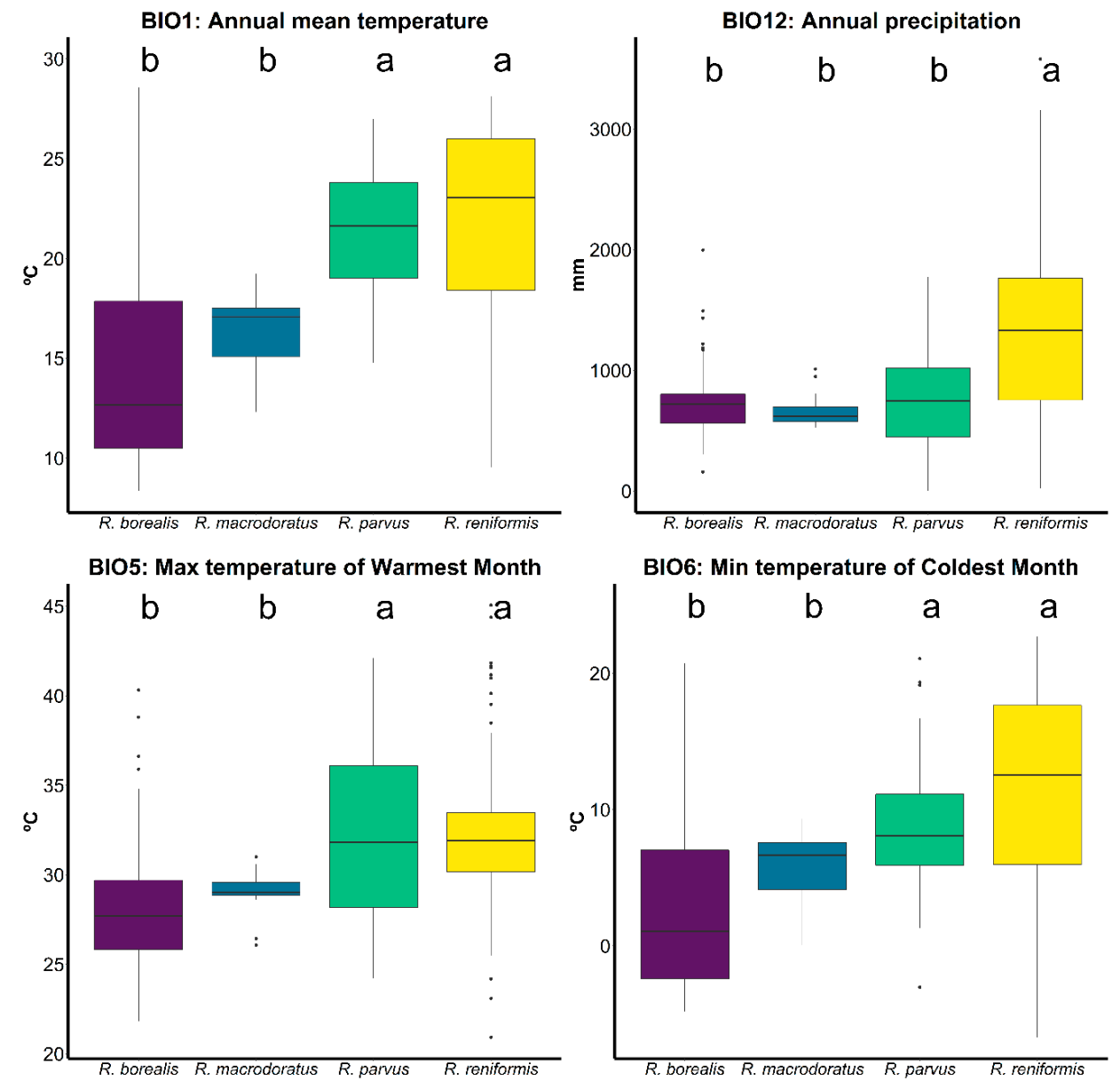

Figure 7. Annual mean temperature, annual precipitation, maximum temperature of warmest month, and minimum temperature of coldest month for Rotylenchulus species with $\geq 3$ reports (each single dot correspond to a species report). The different lowercase letters indicate the differences in each bioclimatic variable between species. They were tested using ANOVA with a level of significance of $p<0.05$. 


\section{Discussion}

The primary objective of this study was to decipher the intraspecific diversity of $R$. borealis and $R$. macrosoma by applying integrative taxonomical approaches on several new unidentified Rotylenchulus populations from Europe, appearing morphological and morphometrically undistinguishable. Additionally, we aimed to provide new insights into the global distribution and climatic requirements of the genus Rotylenchulus.

The resemblance between the mature females of $R$. borealis and $R$. macrosoma, as well as the general similarity between these two species also in their male and immature female forms, host preferences, and host tissue reactions was emphasized by Cohn and Mordechai [18] studying a topotype population of R. macrosoma from olive under growth chamber conditions. Our morphometric studies in this research support that both species do not have major differences in basic morphology or in morphometric informative characters such as immature female body length, stylet length, tail hyaline region, and spicules morphology and morphometry, showing a remarkable example of a close phylogenetic relationship of both species. The results on our new measurements on R. macrosoma immature female paratype specimens from WANECO and USDA nematode collections suggest that the range in stylet length could probably be shorter than that provided in the original description [10], but unfortunately no other paratypes could be studied. The morphometric comparison of an important number of populations from $R$. borealis and $R$. macrosoma exhibited morphometric variation normally expected among populations of the same Rotylenchulus species. The higher values in all of the three main distinguishing morphometric characters between both species were detected in Israel, Crete, and a Spanish population from Huévar del Aljarafe (southern Spain), but these differences do not justify the separation in two different species $[3,4,9,10]$.

In the present study, in which sequence data obtained from $28 S$ and ITS1 rRNA genes and coxI mitochondrial DNA (mtDNA) gene was analyzed, specimens from populations identified as representing $R$. borealis and R. macrosoma from the Netherlands and several European countries, including Israel, respectively, clustered together as a single group. This grouping was well supported by the high bootstrap values in the phylogenetic analysis, thereby supporting the synonymization of R. macrosoma with $R$. borealis, as already emphasized by Cohn and Mordechai [18].

Phylogenetic analyses based on three molecular markers (D2-D3 expansion domains of $28 \mathrm{~S}$ rRNA gene, ITS1 region, and the partial coxI mtDNA) resulted in a general consensus of species phylogenetic positions clustering $R$. borealis population from the Netherlands with $R$. macrosoma from Israel, together with all other $R$. macrosoma populations previously reported in several European countries. These phylogenetic analyses were congruent with those given by previous studies $[3,4,9,16,20]$, and phylogeny of the $28 \mathrm{~S}$ rRNA and ITS regions confirm that $R$. borealis population from the Netherlands is conspecific with $R$. macrosoma from Israel and all other populations from Europe. Our results on $28 \mathrm{~S}$ rRNA phylogeny also suggest that $R$. borealis (MT775429) from New Delhi could not be considered conspecific with $R$. borealis and needs to be revised under integrative taxonomical approaches for confirming its specific status. The genus Rotylenchulus has rRNA genes that exhibit high levels of intraspecific and intra-individual variation $[3,9,16]$. However, they seem functional through the reconstruction of secondary structure models and mutation mapping using $R$. reniformis sequences [3]. Qing et al. [16] suggested that these different sequences are paralogs located in different rRNA clusters or chromosomes and that these tandem arrays may still be expanding in number.

Longer stylet specimens do not seem to be associated with differences in molecular markers (as some Andalusian populations with longer stylet were molecularly associated with other species with shorter stylets) (Figures 3-5). Other characters (body length and hyaline tail region length), as shown in Figure 2, seem to be very variable for African populations of $R$. borealis. Palomares-Rius et al. [4], in a broad molecular study of $R$. borealis (= R. macrosoma), also studied the molecular species separation, with the results showing incongruent results for species separation between Cretan and other European populations 
for $R$. borealis (=R. macrosoma), even with the relatively high molecular differences between both population groups. In our case, the new population of $R$. borealis found in the Netherlands in this study, and the sequence deposited in GenBank from Belgium (MK558206), had an even lower molecular similarity with other former R. macrosoma populations from Crete, Greece, fully supporting our idea of conspecificity.

Thus, the morphological and morphometric results of both species groups, together with the high molecular similarity among ribosomal and mitochondrial genes of both species groups, do not support the validity of $R$. macrosoma as a separate species and give sufficient basis for the synonymization of $R$. macrosoma $\mathrm{n}$. syn. with $R$. borealis. Since the description of $R$. borealis was in 1962 and that of $R$. macrosoma in 1968, the name $R$. borealis has priority over R. macrosoma; thus, $R$. macrosoma is proposed here as a junior synonym of the former.

Climate is a critical environmental determinant of the distribution of plant-parasitic nematodes and a key driver of their reproduction and survival [21]. Temperature, moisture, and availability of host plants are three of the most important factors governing the distribution, spread, and symptom development in plants from plant-parasitic nematodes [21,22], including reniform nematodes. The wide distribution of Rotylenchulus species likely resulted from an exceptionally wide host range, as well as their ability to survive extended periods in a dehydrated state [1]. Anhydrobiotic Rotylenchulus forms have been documented dispersing long distances in dust storms [23]; however, human dispersion through agriculture activities need also to be considered [4]. The influence of annual precipitation on Rotylenchulus spp. distribution suggests that this factor may be not as important as expected. However, the majority of the recorded points have crops with irrigation, and this could change the natural precipitation conditions and importance for these species. In particular, the widespread presence of $R$. borealis in localities at higher latitude in Northern Europe and lower latitude in several central African countries indicated and adaptation to heterogeneous climatic conditions and probably survival strategies for colder and warmer winters and humid to dry soil conditions. Similarly, the cosmopolitan distribution of $R$. parvus can be related to the wide range of temperature reproduction $\left(20\right.$ to $35^{\circ} \mathrm{C}$ ) and survival ( 4 to $35^{\circ} \mathrm{C}$ ), as suggested by Dasgupta and Raski [24]. Climate change could expand $R$. borealis to upper latitudes as climate will warm and this will fulfil the ecological requirements of this species, one of the most adapted to lower temperatures among the four most distributed species ( $R$. borealis, $R$. macrodoratus, R. parvus, and $R$. reniformis). Interestingly, the major diversity of the genus is from sub-Saharan Africa, with the exception of $R$. macrodoratus (Mediterranean distribution) and $R$. leptus (Arabian Peninsula). Siddiqi [25] proposed the idea about the origin of this genus in the Afrotropical (Ethiopian) zoogeographical region, comprising Africa (south of the Sahara); the southern part of the Arabian Peninsula; and various islands, including Madagascar. This idea was reinforced with phylogenetic analysis [3]. However, only three species (R. borealis, R. parvus, and $R$. reniformis) have been able to colonize different continents with wide ecological requirements, as was shown in this research. Additionally, to these ecological requirements for species distribution, other factors such as survival in anhydrobiotic stage or resting eggs could help with the dispersal of this species to other agricultural areas in the world.

In summary, the present study confirmed the synonymy of R. macrosoma with $R$. borealis, and thus the genus comprises 10 valid species. Our data also demonstrate the extraordinary morphological and molecular diversity of $R$. borealis in Europe, Africa, and the Middle East and comprise a paradigmatic example of remarkable flexibility of climatic requirements within reniform nematodes. Nevertheless, despite frequent surveys in different continents of the world, the number of sites studied is still low. Therefore, further surveys are still needed in unsampled geographical areas and climatic conditions, both in plantations and indigenous forests with the aim to identify additional Rotylenchulus species. 


\section{Materials and Methods}

\subsection{Nematode Populations and Morphometric Studies}

One of the authors (G. Karssen) visited the type locality of R. borealis, and the place reported in the original description was lost, i.e., was filled up by new building of houses. Nevertheless, this author detected a new population of $R$. borealis in another location close near the type locality, at Huissen, Betuwe region, the Netherlands. This new population, together with mounted paratypes from of $R$. macrosoma and $R$. borealis from the nematode collections Wageningen Nematode Collection (WANECO; slides WT106, WT107, WT110, WT111, and \#1025 NT and \#1026 NT) and USDA Nematode Collection kindly provided by Dr. Z. A. Handoo (slides T-594p and T-595p), were used for morphological studies.

In addition, some new European reports recently detected and associated with corn and wheat [4] were measured in order to carry out a morphometric comparison with all the measured populations of both species (Tables 2-8). All these populations were compared with the morphometry of all previously studied populations of both species, including a total of 12 populations of $R$. borealis and 16 populations of $R$. macrosoma.

Nematodes were extracted from $500 \mathrm{~cm}^{3}$ of soil by centrifugal flotation [26] method. For morphometric studies, Rotylenchulus specimens were killed and fixed by a hot solution of $4 \%$ formalin $+1 \%$ glycerol, then processed in pure glycerin [27], as modified by De Grisse [28]. The light micrographs and measurements of each nematode population including important diagnostic characteristics (i.e., de Man indices, body length, stylet length, lip region, tail length, etc.) were performed using a Leica DM6 compound microscope with a Leica DFC7000 T digital camera. Nematodes were identified at the species level using an integrative approach combining molecular and morphological techniques to achieve efficient and accurate identification [4,9]. For each nematode population, key diagnostic characters were determined, including body length, stylet length, a ratio (body length/maximum body width), $\mathrm{c}^{\prime}$ ratio (tail length/body width at anus), $\mathrm{V}$ ratio ((distance from anterior end to vulva/body length) $\times 100)$, and o ratio ((distance from stylet base to dorsal pharyngeal opening/body length) $\times 100$ ) [9], and the sequencing of specific DNA fragments (described below) confirmed the identity of the nematode species for each population.

\subsection{DNA Extraction, $P C R$, and Sequencing}

For molecular analyses, in order to ensure that the selected nematodes for extracting DNA are from the same species, we temporary mounted 2 live nematodes from each sample in a drop of $1 \mathrm{M} \mathrm{NaCl}$ containing glass beads (to avoid nematode crushing/damaging specimens) to ensure specimens conformed to the unidentified populations of Rotylenchulus. All necessary morphological and morphometric data by taking pictures and measurements using the above camera-equipped microscope were recorded. This was followed by DNA extraction from a single specimen and polymerase chain reaction (PCR) cycle conditions, as previously described [4,9]. PCR and sequencing of the Dutch population was performed at the Institute for Sustainable Agriculture, Spanish National Research Council (IAS-CSIC) facility, whereas for the Israeli population at Agricultural Research organization (ARO)-Volcani Center, Israel. Several sets of primers were used for PCR. A partial region of the $28 \mathrm{~S}$ rRNA gene including the expansion domains D2 and D3 (D2-D3) was amplified by using the primers D2A (5'-ACAAGTACCGTGAGGGAAAGTTG-3') and D3B ( $5^{\prime}$-TCGGAAGGAACCAGCTACTA-3') [29]. The internal transcribed spacer region (ITS) was amplified using forward primer TW81 (5'-GTTTCCGTAGGTGAACCTGC-3') and reverse primer AB28 (5'-ATATGCTTAAGTTCAGCGGGT $\left.-3^{\prime}\right)$ [30]. The coxI gene was amplified using the primers JB3 (5'-TTTTTTGGGCATCCTGAGGTTTAT-3') and JB5 (5'-AGCACCTAAACTTAAAACATAATGAAAATG-3') [31]. The PCR cycling conditions for the $28 \mathrm{~S}$ rRNA primers were as follows: $94{ }^{\circ} \mathrm{C}$ for $2 \mathrm{~min}$, followed by 35 cycles of $94{ }^{\circ} \mathrm{C}$ for $30 \mathrm{~s}$, an annealing temperature of $55^{\circ} \mathrm{C}$ for $45 \mathrm{~s}$, and $72{ }^{\circ} \mathrm{C}$ for $1 \mathrm{~min}$, and 1 final cycle of $72{ }^{\circ} \mathrm{C}$ for $10 \mathrm{~min}$. The PCR cycling for coxI primers was as follows: $95^{\circ} \mathrm{C}$ for $15 \mathrm{~min}$, 39 cycles at $94{ }^{\circ} \mathrm{C}$ for $30 \mathrm{~s}, 53{ }^{\circ} \mathrm{C}$ for $30 \mathrm{~s}$, and $68^{\circ} \mathrm{C}$ for $1 \mathrm{~min}$, followed by a final extension 
at $72{ }^{\circ} \mathrm{C}$ for $7 \mathrm{~min}$. PCR volumes were adapted to $25 \mu \mathrm{L}$ for each reaction, and primer concentrations were as described in De Ley et al. [29] and Bowles et al. [31]. We used 5x HOT FIREpol Blend Master Mix (Solis Biodyne, Tartu, Estonia) in all PCR reactions. The PCR products were purified after amplification using ExoSAP-IT (Affimetrix, USB products, Kandel, Germany) and used for direct sequencing in both directions with the corresponding primers. Israeli amplification products were cloned before sequencing using pGEM-T easy vector systems (Promega). The resulting products were purified and run in a DNA multicapillary sequencer (Model 3130XL Genetic Analyzer; Applied Biosystems, Foster City, CA, USA), using the BigDye Terminator Sequencing Kit v.3.1 (Applied Biosystems) at the Stab Vida sequencing facility (Caparica, Portugal). The sequence chromatograms of the 2 markers (coxI and D2-D3 expansion segments of 28S rRNA) were analyzed using DNASTAR LASERGENE SeqMan v. 7.1.0. Basic local alignment search tool (BLAST) at the National Center for Biotechnology Information (NCBI) was used to confirm the species identity of the DNA sequences obtained in this study [32]. The newly obtained sequences were deposited in the GenBank database under accession numbers indicated on the phylogenetic trees and in Table 1.

\subsection{Phylogenetic Analysis}

Sequenced genetic markers in the present study (after discarding primer sequences and ambiguously aligned regions) and several Rotylenchulus spp. sequences obtained from GenBank were used for phylogenetic reconstruction (Table 1). Outgroup taxa for each dataset were selected on the basis of previous published studies [3,4,9]. Multiple sequence alignments of the newly obtained and published sequences were made using the Fast Fourier transform-normalized similarity matrix (FFT-NS-2) algorithm of MAFFT v. 7.450 [33]. Sequence alignments were visualized using BioEdit [34] and edited by Gblocks ver. 0.91b [35] in Castresana Laboratory server (http:/ / molevol.cmima.csic.es/castresana/ Gblocks_server.html) using options for a less stringent selection (minimum number of sequences for a conserved or a flanking position: $50 \%$ of the number of sequences +1 ; maximum number of contiguous no conserved positions: 8; minimum length of a block: 5; allowed gap positions: with half).

Phylogenetic analyses of the sequence datasets were based on Bayesian inference (BI) using MRBAYES 3.2.7a [36]. The best-fit model of DNA evolution was calculated with the Akaike information criterion (AIC) of JMODELTEST v. 2.1.7 [37]. The best-fit model, the base frequency, the proportion of invariable sites, and the gamma distribution shape parameters and substitution rates in the AIC were then used in phylogenetic analyses. BI analyses were performed under a general time reversible, with a proportion of invariable sites and a rate of variation across sites (GTR $+\mathrm{I}+\mathrm{G})$ model for D2-D3, ITS1 rRNA, and the partial coxI gene. These BI analyses were run separately per dataset with 4 chains for $2 \times 10^{6}$ generations. The Markov chains were sampled at intervals of 100 generations. Two runs were conducted for each analysis. After discarding burn-in samples of $30 \%$ and evaluating convergence, we retained the remaining samples for more in-depth analyses. The topologies were used to generate a 50\% majority-rule consensus tree. Posterior probabilities (PP) were given on appropriate clades. Trees from all analyses were visualized using FigTree software version v.1.42 [38].

\subsection{Data Collection of Global Distribution of Rotylenchulus spp. and Statistical Analysis}

The species distribution data of Rotylenchulus spp. were exhaustively compiled from the national and regional nematofauna records worldwide from databases (Google Scholar, Web of Sciences, Scopus, and PubMed) and specialized literature (nematological and phytopathological journals) during the period 2020-1940. We selected only those articles satisfying one the following criteria for this review: (1) contained geographical information about the presence and/or abundance of reniform nematodes (Rotylenchulus spp.); (2) contained data on their taxonomy, morphology, molecular identification, ecology, pathogenicity, and provided localities of each population. Articles lacking information about geographic 
coordinates were cross-checked using Quantum GIS v. 3.12.0 [39]. Nevertheless, since $R$. reniformis has been associated with hundreds of crops and native plants in many regions of the world (on the four aforementioned databases we found 9640, 1377, 446, and 189 studies, respectively), only selected reports concerning geographical information were selected, and duplicity of reported localities were not included.

We used bioclimatic predictors (BIOCLIM) based on temperature and precipitation [40] to detect environmental conditions associated with the global distribution of Rotylenchulus spp. and to compare the climate spaces for the different species. Additionally, we plotted the global distribution Rotylenchulus spp. across climate zones on the basis of the type of vegetation [19]. Only species with more than 3 reported populations were plotted in order to assess the range of climatic variables for each species. Species with type locality only or occasional records were omitted.

The analysis on the bioclimatic variables for Rotylenchulus spp. with more than 3 reported populations was concentrated in 18 variables: BIO1 (Annual mean temperature), BIO2 [Mean Diurnal Range (Mean of monthly (max temp-min temp)], BIO3 [Isothermality, $(\mathrm{BIO} 2 / \mathrm{BIO} 7) * 100], \mathrm{BIO} 4$ [Temperature seasonality, (standard deviation * 100)], BIO5 (maximum temperature of the warmest month), BIO6 (minimum temperature of the coldest month), BIO7 [temperature annual range (BIO5-BIO6)], BIO9 (mean temperature of driest quarter), BIO10 (mean temperature of the warmest quarter), BIO 15 (precipitation seasonality, coefficient of variation), and BIO18 (precipitation of the warmest quarter). To detect the influence on Rotylenchulus spp. of the different bioclimatic variables, we used one-way ANOVA among species conducted using the R v. 3.5.1 freeware [41]

Supplementary Materials: The following material is available online at https:/ /www.mdpi.com/22 23-7747/10/1/7/s1. Figure S1. BIOCLIM variables for Rotylenchulus species with $\geq 3$ reports. BIO3 [Isothermality, $(\mathrm{BIO} 2 / \mathrm{BIO} 7) * 100], \mathrm{BIO} 4$ [Temperature seasonality, (SD * 100)], BIO7 [Temperature annual range (BIO5-BIO6)], BIO9 (mean temperature of driest quarter), BIO10 (mean temperature of the warmest quarter), BIO 15 [precipitation seasonality (CV)], BIO17 (precipitation of driest quarter), and BIO18 (precipitation of the warmest quarter).

Author Contributions: Conceptualization, J.E.P.-R., I.C.-C., A.A.-Y., G.L.-R., C.C.-N., S.B.M., G.K., and P.C.; methodology, J.E.P.-R., I.C.-C., A.A.-Y., G.L.-R., C.C.-N., S.B.M., G.K., and P.C.; software, J.E.P.-R., I.C.-C., A.A.-Y., G.L.-R., C.C.-N., and P.C.; analysis, J.E.P.-R., I.C.-C., A.A.-Y., G.L.-R., C.C.-N., and P.C.; resources, J.E.P.-R. and P.C.; writing, J.E.P.-R., I.C.-C., A.A.-Y., C.C.-N., S.B.M., G.K., and P.C. All authors contributed to the final discussion data, and read and approved the final manuscript. All authors have read and agreed to the published version of the manuscript.

Funding: This research was supported by grant RTI2018-095925-A-100 from Ministerio de Ciencia, Innovación y Universidades, Spain, grant 201740E042 “Análisis de diversidad molecular, barcoding, y relaciones filogenéticas de nematodos fitoparásitos en cultivos mediterráneos" from the Spanish National Research Council (C.S.I.C.), and by the Humboldt Research Fellowship for Postdoctoral Researchers awarded to the third author (A.A.-Y.).

Data Availability Statement: The datasets generated during and/or analysed during the current study are available from the corresponding author on reasonable request.

Acknowledgments: We would like to thank J. Martin Barbarroja (IAS-CSIC) for their excellent technical assistance in surveys and management of soil samples, as well as further anonymous reviewers and editors for their effort in reviewing the manuscript and helping improve this study. This research is part of the $\mathrm{PhD}$ project of the second author. The second author is a recipient of a contract from Ministry of Science and Innovation for Predoctoral Researchers in Spain, PRE2019-090206. The third author is a recipient of the Humboldt Research Fellowship for Postdoctoral Researchers at the Helmholtz Centre for Environmental Research-UFZ, Leipzig, Germany. We acknowledge support of the publication fee by the CSIC Open Access Publication Support Initiative through its Unit of Information Resources for Research (URICI).

Conflicts of Interest: The authors declare no conflict of interest. The funders had no role in the design of the study; in the collection, analyses, or interpretation of data; in the writing of the manuscript; or in the decision to publish the results. 


\section{References}

1. Robinson, A.F.; Inserra, R.N.; Caswell-Chen, E.P.; Vovlas, N.; Troccoli, A. Rotylenchulus species: Identification distribution, host ranges, and crop plant resistance. Nematropica 1997, 27, 127-180.

2. Linford, M.B.; Oliveira, J.M. Rotylenchulus reniformis nov. gen., n. sp., a nematode parasite of roots. Proc. Helm. Soc. Wash. 1940, 7 , 35-42.

3. Van den Berg, E.; Palomares-Rius, J.E.; Vovlas, N.; Tiedt, L.R.; Castillo, P.; Subbotin, S.A. Morphological and molecular characterisation of one new and several known species of the reniform nematode, Rotylenchulus Linford \& Oliveira, 1940 (Hoplolaimidae: Rotylenchulinae), and a phylogeny of the genus. Nematology 2016, 18, 67-107.

4. Palomares-Rius, J.E.; Archidona-Yuste, A.; Cantalapiedra-Navarrete, C.; Azpilicueta, A.; Saborido, A.; Tzortzakakis, E.A.; Cai, R.; Castillo, P. New distribution and molecular diversity of the reniform nematode Rotylenchulus macrosoma Dasgupta, Raski and Sher, 1968 (Nematoda: Rotylenchulinae) in Europe. Phytopathology 2020, 110. in press. [CrossRef]

5. Colagiero, M.; Ciancio, A. Climate change and nematodes: Expected effects and perspectives for plant protection. Redia 2011, 94, 113-118.

6. Jones, L.M.; Koehler, A.K.; Trnka, M.; Balek, J.; Challinor, A.J.; Atkinson, H.J.; Urwin, P.E. Climate change is predicted to alter the current pest status of Globodera pallida and G. rostochiensis in the United Kingdom. Glob. Chang. Biol. 2017, 23, 4497-4507. [CrossRef]

7. IPCC. Intergovernmental Panel on Climate Change. 2019. Available online: https://archive.ipcc.ch/index.htm (accessed on 17 September 2020).

8. Bellard, C.; Bertelsmeier, C.; Leadley, P.; Thuiller, W.; Courchamp, F. Impacts of climate change on the future of biodiversity. Ecol. Lett. 2012, 15, 365-377. [CrossRef]

9. Palomares-Rius, J.E.; Cantalapiedra-Navarrete, C.; Archidona-Yuste, A.; Tzortzakakis, E.A.; Birmpilis, I.G.; Vovlas, N.; Subbotin, S.A.; Castillo, P. Prevalence and molecular diversity of reniform nematodes of the genus Rotylenchulus (Nematoda: Rotylenchulinae) in the Mediterranean Basin. Eur. J. Plant Pathol. 2018, 150, 439-455. [CrossRef]

10. Dasgupta, D.R.; Raski, D.J.; Sher, S.A. A revision of the genus Rotylenchulus Linford and Oliveira, 1940 (Nematoda: Tylenchidae). Proc. Helminthol. Soc. Wash. 1968, 35, 169-192.

11. Loof, P.A.A.; Oostenbrink, M. Rotylenchulus borealis n. sp. with a key to the species of Rotylenchulus. Nematologica 1962, 7, 83-90.

12. Germani, G. Morphological and biometrical characters of three West-African species of Rotylenchulus Linford \& Oliveira, 1940 (Nematoda: Tylenchida). Rev. Nématol. 1978, 1, 241-250.

13. Castillo, P.; Vovlas, N.; Troccoli, A. The reniform nematode, Rotylenchulus macrosoma, infecting olive in southern Spain. Nematology 2003, 5, 23-29. [CrossRef]

14. Niragire, I. Morphological and Molecular Characterization of Plant-Parasitic Nematodes of Potato (Solanum tuberosum I.) in Rwanda. Master's Thesis, University of Ghent, Ghent, Belgium, 2018; p. 44.

15. Singh, P.R.; Kashando, B.E.; Couvreur, M.; Karssen, G.; Bert, W. Plant-parasitic nematodes associated with sugarcane in Kilimanjaro, Tanzania. J. Nematol. 2020, 52, e2020-59. [CrossRef]

16. Qing, X.; Bik, H.; Yergaliyev, T.M.; Gu, J.; Fonderie, P.; Brown-Miyara, S.; Szitenberg, A.; Bert, W. Widespread prevalence but contrasting patterns of intragenomic rRNA polymorphisms in nematodes: Implications for phylogeny, species delimitation and life history inference. Mol. Ecol. Resour. 2020, 20, 318-332. [CrossRef]

17. Lišková, M.; Troccoli, A.; Vovlas, N.; Sasanelli, N. On the occurrence of Rotylenchulus borealis in the Slovak Republic. Helminthologia 2002, 39, 165-167.

18. Cohn, E.; Mordechai, M.M. Morphology and parasitism of the mature female of Rotylenchulus macrosomus. Rev. Nématol. 1988, 11, 385-389.

19. Kottek, M.; Grieser, J.; Beck, C.; Rudolph, B.; Rubel, F. World map of the Köppen-Geiger climate classification updated. Meteorol. Z. 2006, 15, 259-263. [CrossRef]

20. Riascos-Ortiz, D.; Mosquera-Espinosa, A.T.; De Agudelo, F.V.; de Oliveira, C.M.G.; Muñoz-Flórez, J.E. Morpho-molecular characterization of Colombian and Brazilian populations of Rotylenchulus associated with Musa spp. J. Nematol. 2019, 51, e2019-47. [CrossRef]

21. Thakur, M.P.; Tilman, D.; Purschke, O.; Ciobanu, M.; Cowles, J.; Isbell, F.; Wragg, P.D.; Eisenhauer, N. Climate warming promotes species diversity, but with greater taxonomic redundancy, in complex environments. Sci. Adv. 2017, 3. [CrossRef]

22. Neher, D.A. Ecology of plant and free-living nematodes in natural and agricultural soil. Annu. Rev. Phytopathol. 2010, 48, 371-394. [CrossRef]

23. Gaur, H.S. Dissemination and mode of survival of nematodes in dust storms. Ind. J. Nematol. 1988, 18, 84-98.

24. Dasgupta, D.R.; Raski, D.J. The biology of Rotylenchulus parvus. Nematologica 1968, 14, 429-440.

25. Siddiqi, M.R. Tylenchida Parasites of Plants and Insects, 2nd ed.; CABI Publishing: Wallingford, UK, 2000.

26. Coolen, W.A. Methods for extraction of Meloidogyne spp. and other nematodes from roots and soil. In Root-Knot Nematodes (Meloidogyne species). Systematics, Biology, and Control; Lamberti, F.F., Taylor, C.E., Eds.; Academic Press: London, UK, 1979; pp. 317-329.

27. Seinhorst, J.W. Killing nematodes for taxonomic study with hot f.a. 4:1. Nematologica 1966, 12, 178. [CrossRef]

28. De Grisse, A.T. Redescription ou modification de quelques techniques utilisées dans l'étude des nematodes phytoparasitaires. Meded. Rijksfak. Landbouwet. 1969, 34, 351. 
29. De Ley, P.; Felix, M.A.; Frisse, L.A.; Nadler, S.; Sternberg, P.; Thomas, W. Molecular and morphological characterisation of two reproductively isolated species with mirror-image anatomy (Nematoda: Cephalobidae). Nematology 1999, 1, 591-612. [CrossRef]

30. Subbotin, S.A.; Vierstraete, A.; De Ley, P.; Rowe, J.; Waeyenberge, L.; Moens, M.; Vanfleteren, J.R. Phylogenetic relationships within the cyst-forming nematodes (Nematoda, Heteroderidae) based on analysis of sequences from the ITS regions of ribosomal DNA. Mol. Phylogenet. Evol. 2001, 21, 1-16. [CrossRef]

31. Bowles, J.; Blair, D.; McManus, D.P. Genetic variants within the genus Echinococcus identified by mitochondrial DNA sequencing. Mol. Biochem. Parasitol. 1992, 54, 165-174. [CrossRef]

32. Altschul, S.; Gish, W.; Miller, W.; Myers, E.W.; Lipman, D.J. Basic local alignment search tool. J. Mol. Biol. 1990, 215, 403-410. [CrossRef]

33. Katoh, K.; Rozewicki, J.; Yamada, K.D. MAFFT online service: Multiple sequence alignment, interactive sequence choice and visualization. Brief. Bioinform. 2019, 20, 1160-1166. [CrossRef]

34. Hall, T.A. BioEdit: A user-friendly biological sequence alignment editor and analysis program for windows 95/98/NT. Nucleic Acids Symp. Ser. 1999, 41, 95-98.

35. Castresana, J. Selection of conserved blocks from multiple alignments for their use in phylogenetic analysis. Mol. Biol. Evol. 2000, 17, 540-552. [CrossRef] [PubMed]

36. Ronquist, F.; Huelsenbeck, J.P. MRBAYES 3: Bayesian phylogenetic inference under mixed models. Bioinformatics 2003, 19, 1572-1574. [CrossRef] [PubMed]

37. Darriba, D.; Taboada, G.L.; Doallo, R.; Posada, D. jModelTest 2: More models, new heuristics and parallel computing. Nat. Methods 2012, 9, 772. [CrossRef] [PubMed]

38. Rambaut, A. FigTreev1.4.2. A Graphical Viewer of Phylogenetic Trees. 2014. Available online: http://tree.bio.ed.ac.uk/software/ figtree/ (accessed on 20 October 2020).

39. QGIS Development Team. QGIS Geographic Information System. Open Source Geospatial Foundation Project. 2020. Available online: http:/ / qgis.osgeo.org (accessed on 12 November 2020).

40. Fick, S.E.; Hijmans, R.J. WorldClim 2: New $1 \mathrm{~km}$ spatial resolution climate surfaces for global land areas. Int. J. Climatol. 2017, 37, 4302-4315. [CrossRef]

41. R_Core_Team. R: A language and environment for statistical computing. In R Foundation for Statistical Computing; R Foundation: Vienna, Austria, 2018; Available online: https:/ / www.R-project.org/ (accessed on 6 October 2020). 